\title{
SPECTROSCOPIC CHARACTERIZATION AND CYTOTOXIC ACTIVITY OF NEW METAL COMPLEXES DERIVED FROM (1E, N'Z, N'Z)-N',N'-BIS(2- HYDROXYBENZYLIDENE)-2-(NAPHTHALEN-1-YLOXY) ACETOHYDRAZONOHYDRAZIDE.
}

\author{
A. S. El-Tabl', M. M. Abd-El Wahed², M. A. Wahba ${ }^{3}$ \\ and M. A. Shebl ${ }^{1}$ \\ ${ }^{1}$ Department of Chemistry, Faculty of Science, El-Menoufia University, Shebin El- Kom, Egypt. \\ ${ }^{2}$ Department of Pathology, Faculty of Medicine, El-Menoufia University, Shebin El- Kom, Egypt. \\ ${ }^{3}$ Department of Inorganic Chemistry, National Research Center, Dokki, Giza, Egypt.
}

\begin{abstract}
Spectroscopic (IR, ${ }^{1} \mathrm{H}$-NMR, UV-visible, mass and ESR spectra) and structural studies of the ligand (1E, N'Z, N'Z)-N', N'-bis (2-hydroxybenzylidene)-2-(naphthalen-1-yloxy) acetohydrazonohydrazide $\left(\mathrm{H}_{2} \mathrm{~L}\right)$ and its metal complexes are reported. The magnetic properties and thermal analyses (DTA and TGA) were also carried out. The IR spectra of the prepared complexes suggested that, the ligand adopted either a bidentate or a tetradentate fashion, bonding to the metal ion through the azomethine nitrogens and the two phenolic oxygen atoms (ONNO). Electronic spectra and magnetic susceptibility measurements revealed an octahedral geometry for all complexes except sliver(I) complex (6), copper(II) complex (7) and cobalt(II) complex (11). The elemental analyses and mass spectral data have justified the $M L, M_{2}$ and $M_{3} L$ composition of the complexes. The ESR spectra of copper(II) complexes (5), (7), (12) and (16), showed an axial type $\left(\mathrm{dx}^{2}-\mathrm{y}^{2}\right)$ ground state with a covalent bond character and also support the suggested structures of complexes. The cytotoxicity of the ligand and its metal complexes were investigated and discussed.
\end{abstract}

Indexing terms/Keywords:-Cytotoxic activity; hydrazine complexes; spectral and magnetic studies.

\section{Council for Innovative Research}

Peer Review Research Publishing System

\section{Journal: Journal of Advances in Chemistry}

Vol. 11, No. 9

www.cirjac.com

editorjaconline@gmail.com, editor@cirjac.com 


\section{INTRODUCTION}

New synthetic compounds with novel mechanism of action have become an important task to cope with drug resistance problems. Schiff bases have largely been recognized as useful building blocks in the synthesis of biologically important compounds [1-3]. Considerable studies have been reported regarding their biological activities as anticancer, antibacterial, antifungal, and herbicidal activities [4-8]. However, many organic drugs require interaction with metals in order to enhance their activity. They interact with metals at their target site or during their metabolism or disturb the balance of metal ion uptake and distribution in cells and tissues. Understanding these interactions helps a lot in synthesizing of influential metallo-pharmaceuticals and implementation of new co-therapies. Metal complexes have unique properties enhancing their bioactivity. An important property is the ability of metals to form positively charged ions in aqueous solutions that can bind to negatively charged biological molecules [9-12]. The high electron affinity of metal ions can significantly polarize groups that are coordinated to them, leading to the generation of hydrolysis reactions [10]. Furthermore, metal ions also has the ability to coordinate ligands in a three dimensional configuration, thus allowing functionalization of groups that can be tailored to defined molecular targets [13,14,7]. Much concern has been drawn toward hydrazine and their metal complexes due to their biological activities as fungicides [15,16]. bactericides [17], analgesic and anti-inflammatory [18], antioxidant [19,20], antitumor [21-23] and insecticidal [24]. Literature survey on structural behavior of hydrazine complexes reveals some interesting features of its coordination behavior. As a ligand, hydrazine offers the possibility of different modes of coordination towards transition metal ions. It can function as a monodentate and or bridging bidentate ligand $[2,25]$. Reactions of hydrazines with complexes containing multiple bonds can give rise to complexes containing coordinated imido-, diazenido- and nitrido-ligands [26-28]. In view of the above facts, this article aimed to synthesize and identify new metal complexes derived from a hydrazine Schiff base ligand. The coordination behavior of the ligand towards metals ions has been investigated via variety of physicochemical techniques. The cytotoxic activity of the ligand as well as its metal complexes was tested against human colon cancer cells (HCT-116 cell line) and hepatocellular carcinoma (HEPG-2 cell line) comparing with standard drug Vinblastine. Furthermore, the antimicrobial activity of some metal complexes against Aspergillus fumigates, Candida albicans, Streptococcus pneumonia, Bacillis subtilis, Pseudomonas aeruginosa and Escherichia coli was also investigated.

\section{EXPRIMENTAL}

\subsection{Materials}

All reagents employed for the preparation of the ligand and its complexes were of the analytical grade available and used without further purification. Metal salts were provided from Sigma-Aldrich Company. 1-naphthol (Assay $99 \%)$ and ethylchloroacetate (Assay $99 \%$ ), hydrazine hydrate (Assay $\geq 99.99 \%$ ), 2-hydroxy benzaldehyde (Assay $\geq 98 \%$ ), DMSO (Assay $99.7 \%$ ) and absolute ethanol (Assay $\geq 99.8 \%$ ) were also obtained from Sigma-Aldrich Company.

\subsection{Instrumentation and measurements}

$\mathrm{C}, \mathrm{H}, \mathrm{N}$ and $\mathrm{Cl}$ were analysed at the Microanalytical center, Cairo University, Egypt. Standard analytical method (gravimetric) was used to determine the metal ion content [29-31]. FT-IR spectra of the ligand and its metal complexes were measured using $\mathrm{KBr}$ discs by a Jasco FT/IR 300E Fourier transform infrared spectrophotometer covering the range $400-4000 \mathrm{~cm}^{-1}$. Electronic spectra in the 200-900 nm regions were recorded on a Perkin-Elmer 550 spectrophotometer. The thermal analyses (DTA and TGA) was carried out on a Shimadzu DT-30 thermal analyzer from room temperature to $800^{\circ} \mathrm{C}$ at a heating rate of $10^{\circ} \mathrm{C} / \mathrm{min}$. Magnetic susceptibilities were measured at $25^{\circ} \mathrm{C}$ by the Gouy method using mercuric tetrathiocyanatocobaltate(II) as the magnetic susceptibility standard. Diamagnetic corrections were estimated from Pascal's constant [32]. The magnetic moments were calculated from the equation:

$$
\mu_{\text {eff. }}=2.84 \sqrt{\chi_{M}^{\text {corr }} \cdot T}
$$

The molar conductance of $10^{-3} \mathrm{M}$ solution of the complexes in DMSO was measured at $25^{\circ} \mathrm{C}$ with a Bibby conductometer type $\mathrm{MCl}$. The resistance measured in ohms and the molar conductivities were calculated according to the equation:

$$
\Lambda_{\mathrm{M}}=\mathrm{V}^{*} \mathrm{~K}^{*} \mathrm{~g} / \mathrm{M}_{\mathrm{w}}{ }^{*}
$$

Where: $\mathrm{M}=$ molar conductivity $/{ }^{-1} \mathrm{~cm}^{2} \mathrm{~mol}^{-1}, \mathrm{~V}=$ volume of the complex solution $/ \mathrm{ml}, \mathrm{K}=$ cell constant $\left(0.92 / \mathrm{cm}^{-1}\right), \mathrm{Mw}=$ molecular weight of the complex, $g=$ weight of the complex/g, =resistance/. ${ }^{1} \mathrm{H}-\mathrm{NMR}$ spectra were obtained on BRUKER $400 \mathrm{MHz}$ spectrometers. Mass spectra were recorded using GC/MS Shimadzu 5050 QA mass spectrometer. Chemical shifts (ppm) are reported relative to TMS. ESR measurements of solid complexes at room temperature were made using a Varian E-109 spectrophotometer with DPPH as a standard material. TLC is used to confirm the purity of the compounds.

\subsection{Synthesis of the ligand}

The ligand $\left[\mathrm{H}_{2} \mathrm{~L}\right]$ was prepared by a three-step reactions (Figure 1). The first one involved addition of equimolar amount of 1 -naphthole $(10 \mathrm{~g}, 1.0 \mathrm{~mol})$, to ethylchloroacetate $(7.38 \mathrm{ml}, 0.1 \mathrm{~mol})$ in the presence of $\mathrm{KOH}(4.5 \mathrm{~g}, 0.10 \mathrm{~mol})$ in $50 \mathrm{~cm}$ of absolute ethanol. The mixture was refluxed on water bath for 6 hours and the formed precipitate was filtered off, washed with water, dried and recrystallized from ethanol to afford ethyl (1-naphthyloxy) acetate (I). The second step includes mixing equimolar amount of ethyl (1-naphthyloxy) acetate (I) $(6.5 \mathrm{~g}, 0.01 \mathrm{~mol})$ with hydrazine hydrate $(2.7 \mathrm{ml}, 0.02 \mathrm{~mol})$ in $50 \mathrm{~cm}^{3}$ of absolute methanol. The solution was refluxed with stirring for 4 hours, and the formed yellow product was filtrated off, washed with water, and dried to give pure needle shaped crystals of 2-(naphthalene-1-yloxy) acetohydrazide 
(II). The final step involved addition of an equimolar amount of 2-(naphthalene-1-yloxy) acetohydrazide (II) (5.0 g, 0.01 mol) to 2-hydroxy benzaldehyde $(5.6 \mathrm{~g}, 0.01 \mathrm{~mol})$ in $50 \mathrm{~cm}^{3}$ of absolute methanol. The mixture was refluxed with continuous stirring for 3 hours. After cooling, the solvent was removed under reduced pressure to give the ligand $\left[\mathrm{H}_{2} \mathrm{~L}\right]$, (1E, N'Z, N'Z)-N', N'-bis (2-hydroxybenzylidene)-2-(naphthalene-1-yloxy) acetohydrazono- hydrazide.<smiles>CCOC(=O)CCl</smiles>

Ligand $\left[\mathrm{H}_{2} \mathrm{~L}\right]$

(1E,N'Z,N'Z)-N',N'-bis(2-hydroxybenzylidene)-2-(naphthalen-1-yloxy)acetohydrazonohydrazide

Figure 1: Synthesis of the ligand $\left[\mathrm{H}_{2} \mathrm{~L}\right]$

\subsubsection{Preparation of complexes (2)-(19)}

To the ligand (1) $(1.0 \mathrm{~g}, 0.002 \mathrm{~mol})$ in ethanol $\left(50 \mathrm{~cm}^{3}\right)$ was added ethanolic solution of $(0.568 \mathrm{~g}, 0.002 \mathrm{~mol})$ of $\mathrm{Co}(\mathrm{OAc})_{2} .4 \mathrm{H}_{2} \mathrm{O}$ (1L:1M), complex (2), $(0.898 \mathrm{~g}, 0.003 \mathrm{~mol})$ of $\mathrm{NiSO}_{4} \cdot 6 \mathrm{H}_{2} \mathrm{O},(2 \mathrm{~L}: 3 \mathrm{M})$, complex (3), to the ligand $(1.0 \mathrm{~g}, 0.002 \mathrm{~mol})$ in ethanol $\left(50 \mathrm{~cm}^{3}\right)$ was added $(0.567 \mathrm{~g}, 0.002 \mathrm{~mol}) \mathrm{Ni}(\mathrm{OAc})_{2} \cdot 4 \mathrm{H}_{2} \mathrm{O},(1 \mathrm{~L}: 1 \mathrm{M})$, complex (4), to the ligand $(1.0 \mathrm{~g}, 0.002 \mathrm{~mol})$ in ethanol $\left(50 \mathrm{~cm}^{3}\right)$ was added $(0.414$ $\mathrm{g}, 0.002 \mathrm{~mol}$ ) of $\mathrm{Cu}(\mathrm{OAc})_{2},(1 \mathrm{~L}: 1 \mathrm{M})$, complex (5), $(0.774 \mathrm{~g}, 0.004 \mathrm{~mol})$ of $\mathrm{Ag}\left(\mathrm{NO}_{3}\right),(1 \mathrm{~L}: 2 \mathrm{M})$, complex (6), $(0.459 \mathrm{~g}, 0.003 \mathrm{~mol})$ of $\mathrm{CuCl}$, (2L:3M), complex (7), (0.951g, $0.003 \mathrm{~mol}$ ) of $\mathrm{FeSO}_{4} \cdot 7 \mathrm{H}_{2} \mathrm{O},(2 \mathrm{~L}: 3 \mathrm{M})$, complex (8), (0.432 g, $\left.0.001 \mathrm{~mol}\right)$ of $\mathrm{Pb}(\mathrm{OAc})_{2}$, (2L:1M), complex (9), $(0.279 \mathrm{~g}, 0.001 \mathrm{~mol})$ of $\mathrm{Mn}(\mathrm{OAc})_{2} .4 \mathrm{H}_{2} \mathrm{O},(2 \mathrm{~L}: 1 \mathrm{M})$, complex (10), $(0.529 \mathrm{~g}, 0.003 \mathrm{~mol})$ of $\mathrm{CoSO}_{4},(2 \mathrm{~L}: 3 \mathrm{M})$, complex (11), (0.551 g , $0.002) \mathrm{Cu}\left(\mathrm{NO}_{3}\right)_{2} \cdot 3 \mathrm{H}_{2} \mathrm{O},(1 \mathrm{~L}: 1 \mathrm{M})$, complex (12), $(0.284 \mathrm{~g}, 0.001 \mathrm{~mol})$ of $\mathrm{Co}(\mathrm{OAc})_{2} \cdot 4 \mathrm{H}_{2} \mathrm{O},(2 \mathrm{~L}: 1 \mathrm{M})$, complex (13), $(0.304 \mathrm{~g}, 0.001 \mathrm{~mol})$ of $\mathrm{Cd}(\mathrm{OAc})_{2} .2 \mathrm{H}_{2} \mathrm{O},(2 \mathrm{~L}: 1 \mathrm{M})$, complex $(14),(0.500 \mathrm{~g}, 0.002 \mathrm{~mol})$ of $\mathrm{Zn}(\mathrm{OAc})_{2} .2 \mathrm{H}_{2} \mathrm{O}$, (1L:1M), complex (15), $(0.853 \mathrm{~g}, 0.003 \mathrm{~mol})$ of $\mathrm{CuSO}_{4} .5 \mathrm{H}_{2} \mathrm{O}$, (2L:3M), complex (16), $(0.363 \mathrm{~g}, 0.001 \mathrm{~mol})$ of $\mathrm{Hg}(\mathrm{OAc})_{2},(2 \mathrm{~L}: 1 \mathrm{M})$, complex (17), $(0.304 \mathrm{~g}, 0.001 \mathrm{~mol})$ of $\mathrm{Sr}(\mathrm{Cl})_{2} .6 \mathrm{H}_{2} \mathrm{O}$, $(2 \mathrm{~L}: 1 \mathrm{M})$, complex (18), $(0.435 \mathrm{gm}, 0.001 \mathrm{~mol})$ of $\mathrm{Ti}\left(\mathrm{C}_{2} \mathrm{H}_{3} \mathrm{O}_{2}\right)_{3},(2 \mathrm{~L}: 1 \mathrm{M})$, complex (19), The mixture was refluxed with stirring for 2-3 hrs, depending on the nature of the metal ion and the anion. When the precipitate appeared, it was removed by filteration, washed with ethanol and dried in vacuo over $\mathrm{p}_{4} \mathrm{O}_{10}$. Analytical data are given in Table 1.

\subsection{Biological activity}

\subsubsection{Cytotoxic activity}

Evaluation of the cytotoxic activity of the ligand and its metal complexes was carried out in the Pathology Laboratory, Pathology Department, Faculty of Medicine, El-Menoufia University, Egypt. The evaluation process was carried out in vitro using the Sulfo-Rhodamine-B-stain (SRB) assay published method [33]. Cells were plated in 96-multiwell plate $\left(10^{4} \mathrm{cells} /\right.$ well $)$ for $24 \mathrm{hrs}$. before treatment with the complexes to allow attachment of cell to the wall of the plate. Different concentrations of the compounds under test in $\operatorname{DMSO}(0,5,12.5,25$ and $50 \mu \mathrm{g} / \mathrm{ml})$ were added to the cell monolayer, triplicate wells being prepared for each individual dose. Monolayer cells were incubated with the complexes for 48 hrs at $37^{\circ} \mathrm{C}$ and under $5 \% \mathrm{CO}_{2}$. After $48 \mathrm{hrs}$.cells were fixed, washed and stained with Sulfo-Rhodamine-B-stain. Excess stain was wash with acetic acid and attached stain was recovered with Tris EDTA buffer. Color intensity was measured in an ELISA reader. The relation between surviving fraction and drug concentration is plotted to get the survival curve for each tumor cell line after addition the specified compound. 


\section{Results and discussion}

The complexes are colored, stable in air; they are soluble in polar solvents such as DMF and DMSO whereas they are insoluble in $\mathrm{H}_{2} \mathrm{O}$, ethanol, $\mathrm{CHCl}_{3}$ and benzene. All the complexes are non-electrolytes. The elemental analyses, spectral data [Tables 1-5] and thermal analyses [Table 6] are compatible with the proposed structures [Figure 2]. Many attempts were made to grow diffractable crystals, but unfortunately no crystal has been obtained until now.<smiles>Oc1ccccc1/C=N\N=C(/COc1cccc2ccccc12)N/N=C\c1ccccc1O</smiles>

$\left(1 E, N^{\prime} Z, N^{\prime} Z\right)-N^{\prime}, N^{\prime}$-bis(2-hydroxybenzylidene)-2-(naphthalen-1-yloxy)acetohydrazonohydrazide

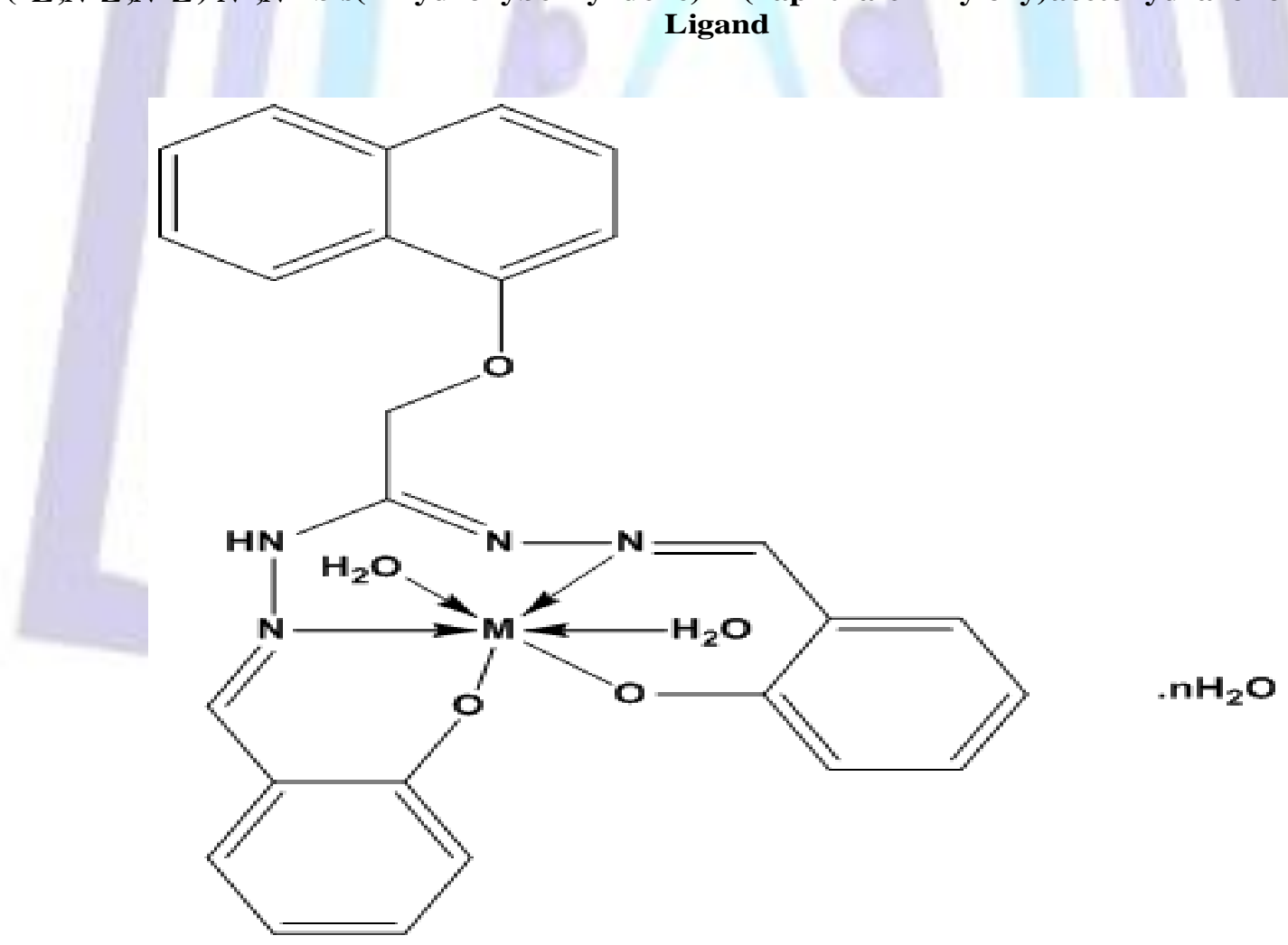

$\begin{array}{lll}\text { Complex (2) } & M=C o & n=1 \\ \text { Complex (4) } & M=N i & n=2 \\ \text { Complex (5) } & M=C u & n=2\end{array}$ 


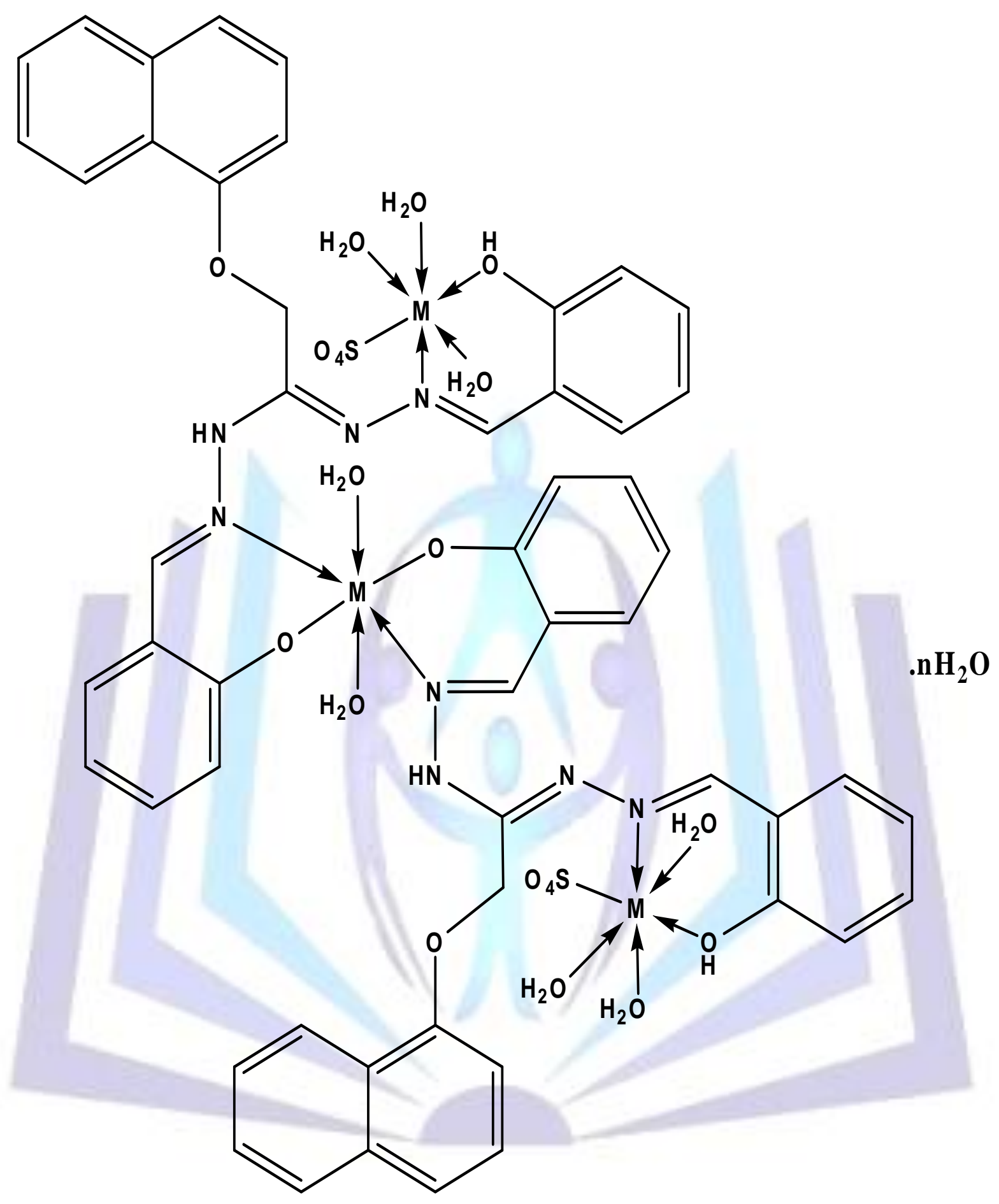

Complex (3) $\quad \mathrm{M}=\mathrm{Ni} \quad \mathrm{n}=2$

Complex (8) $\mathrm{M}=\mathrm{Fe} \quad \mathrm{n}=1$ 

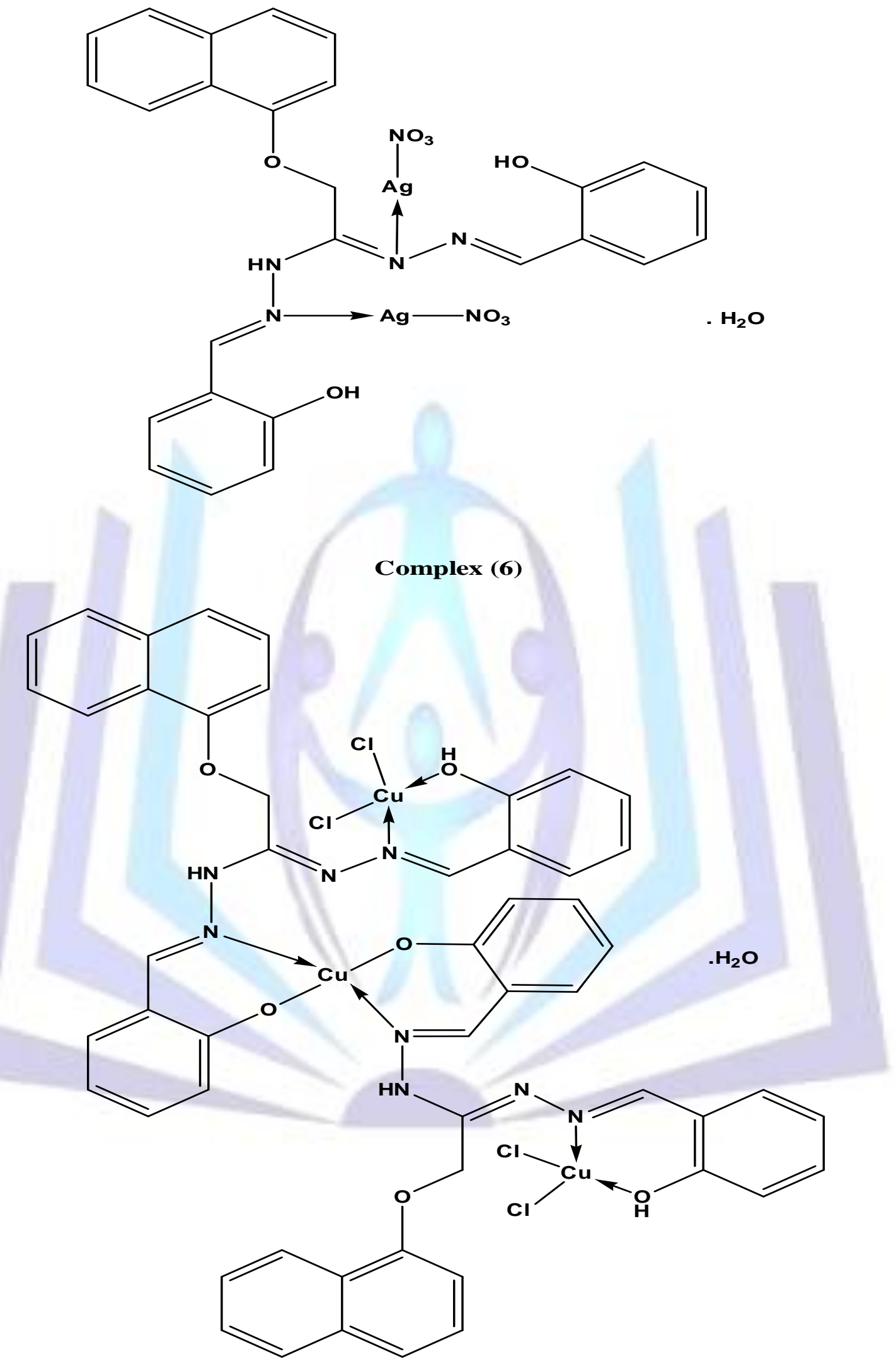

Complex (7) 


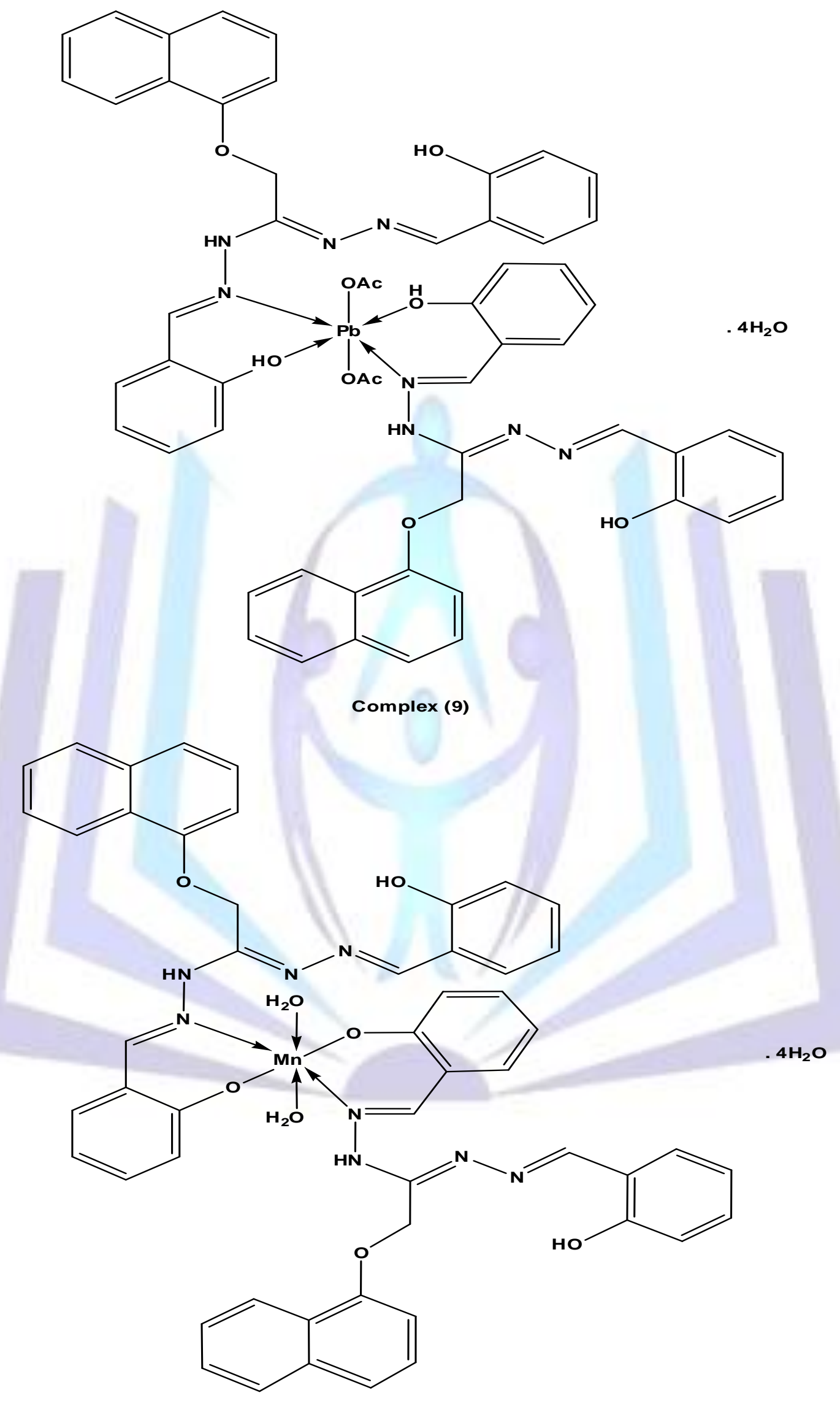

Complex (10) 


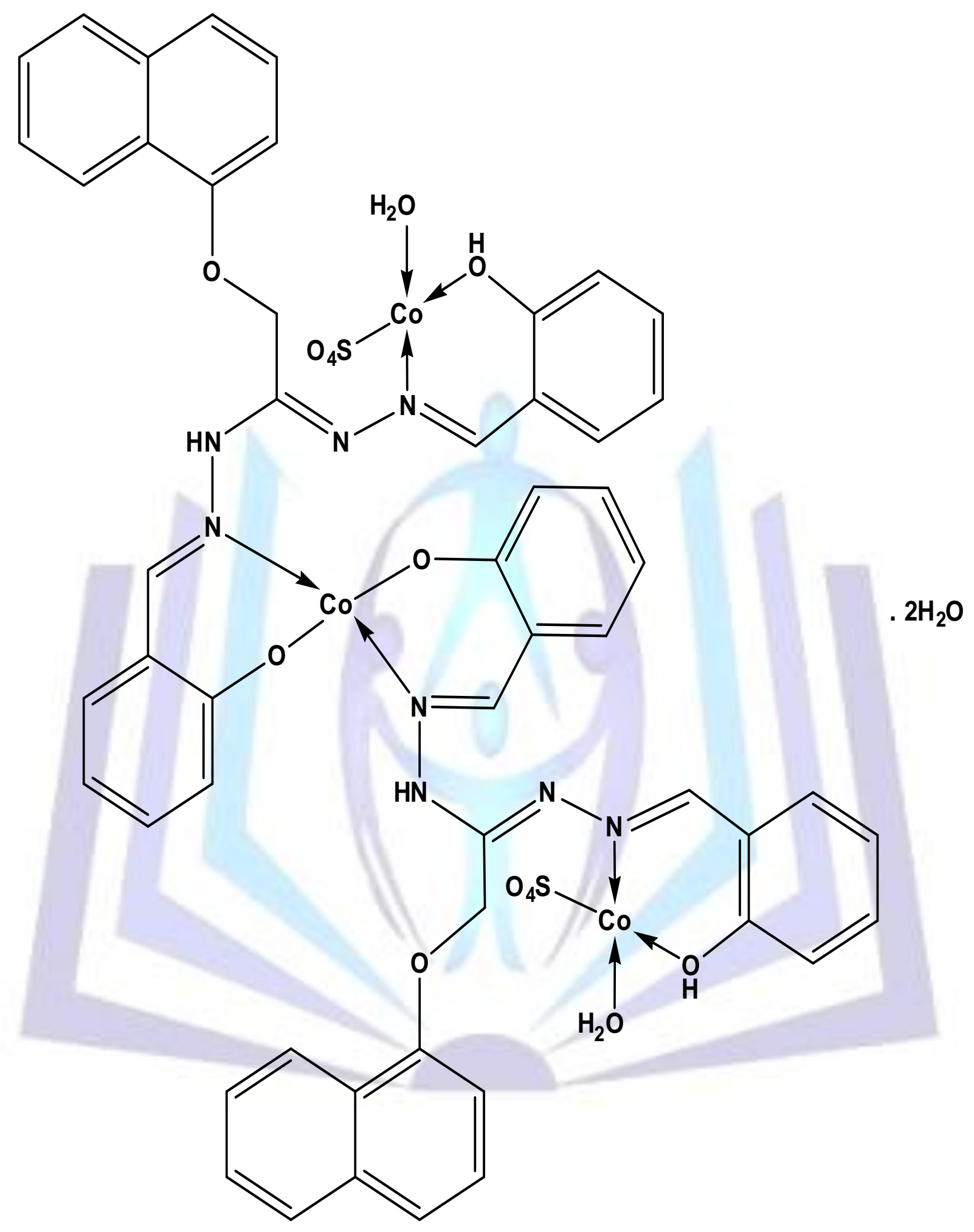

Complex (11) 


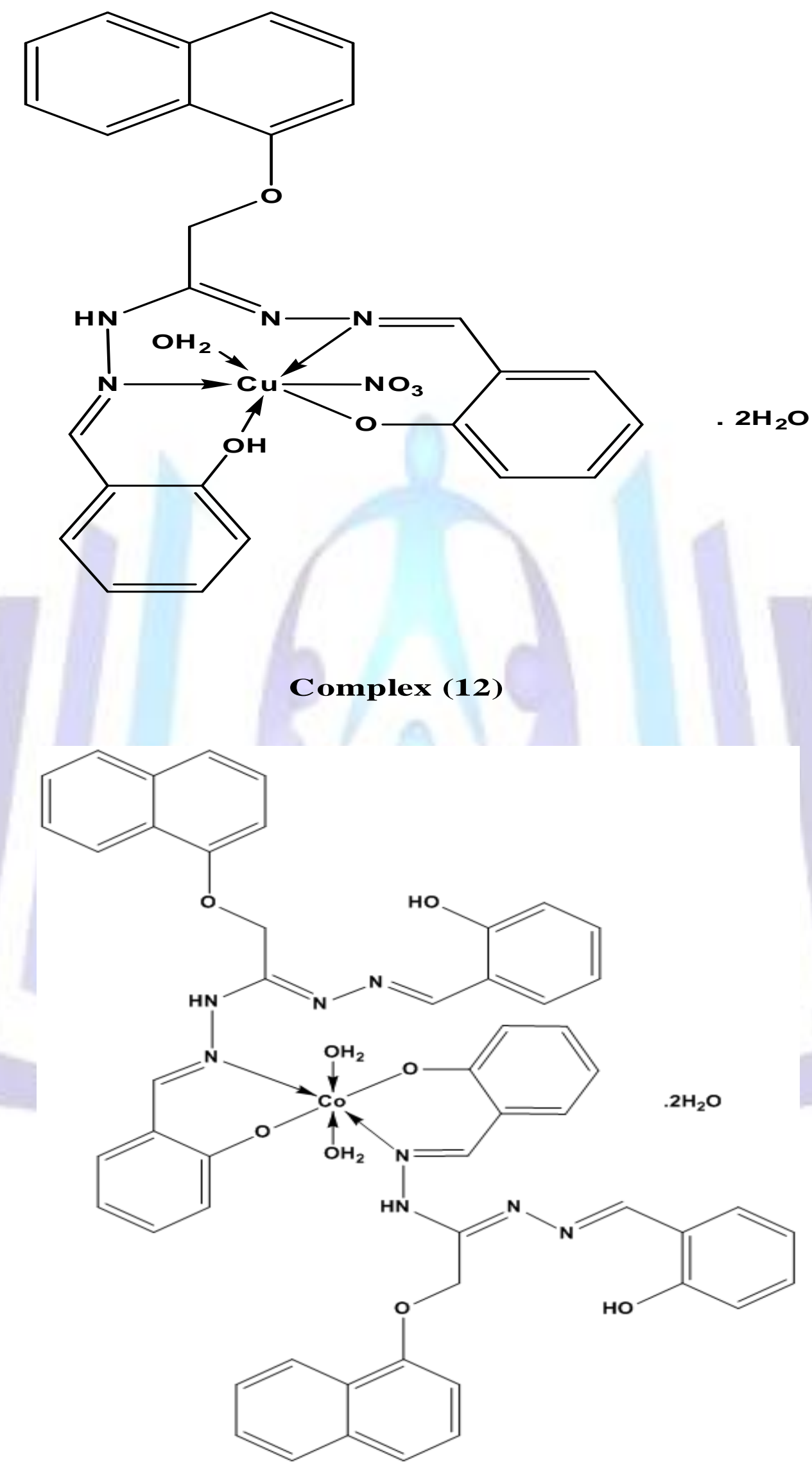

Complex (13) 

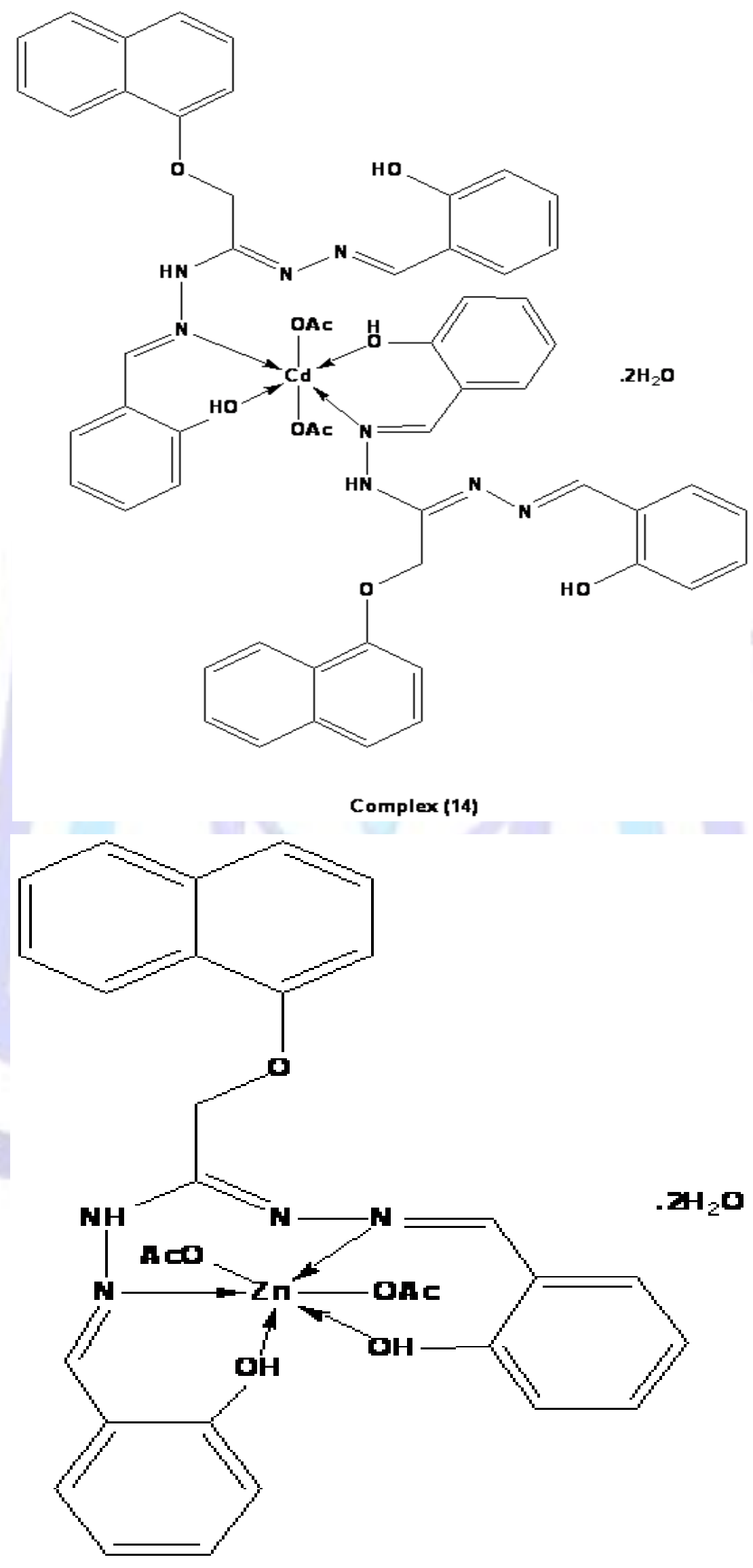

Complex (15) 


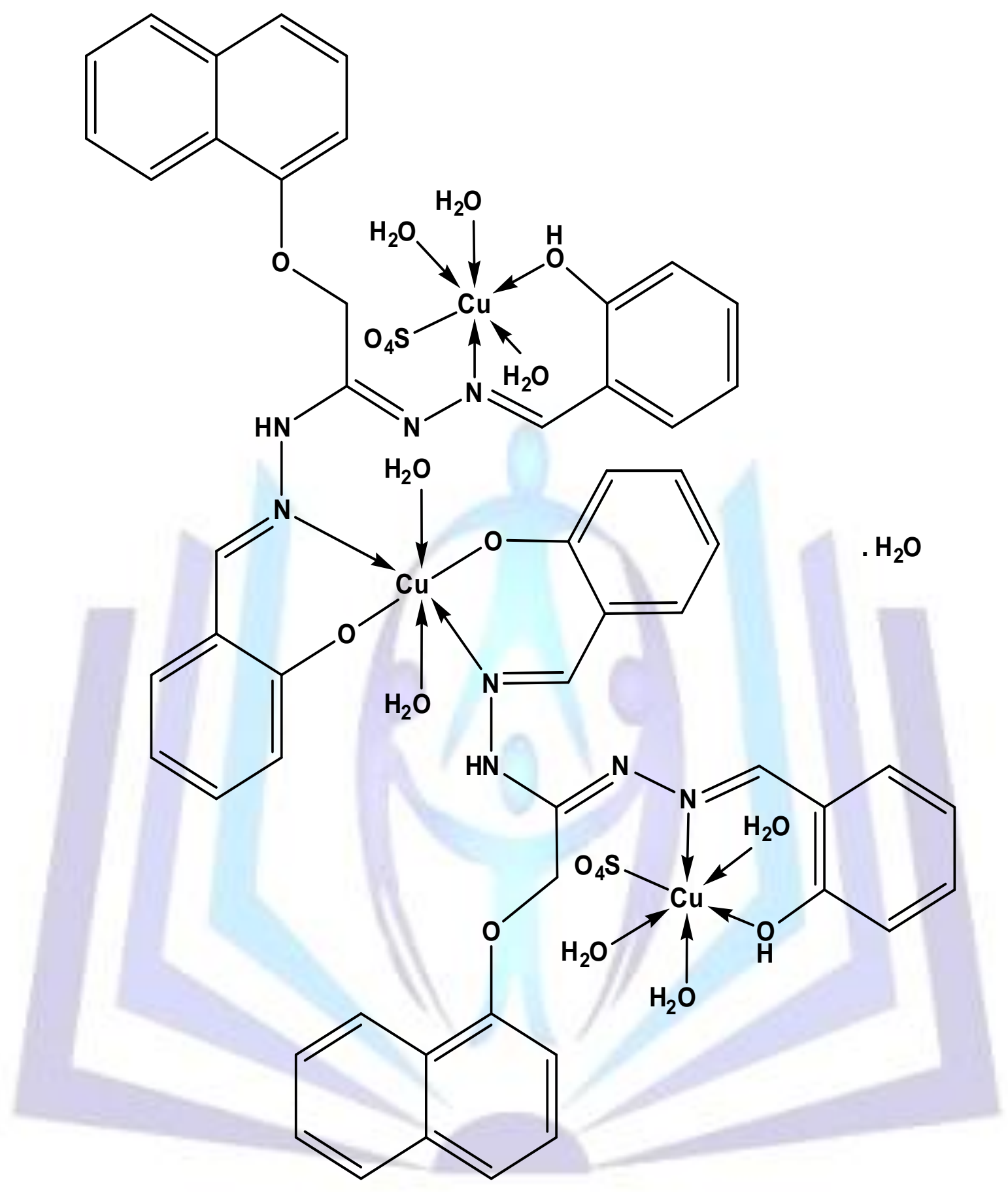

Complex (16) 


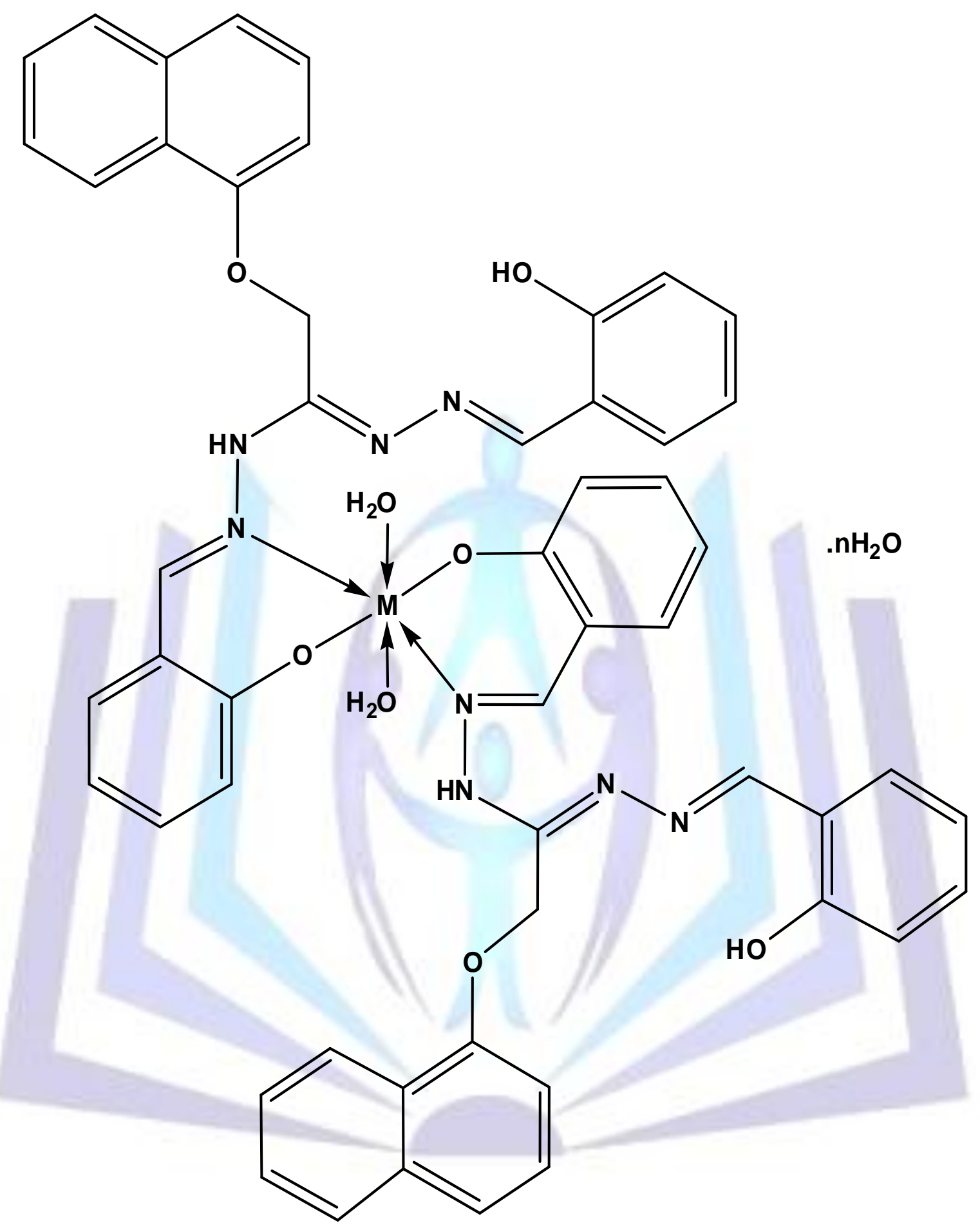

Complex (17) $\quad M=H g \quad n=1$

Complex (18) $\mathrm{M}=\mathrm{Sr} \quad \mathrm{n}=4$ 


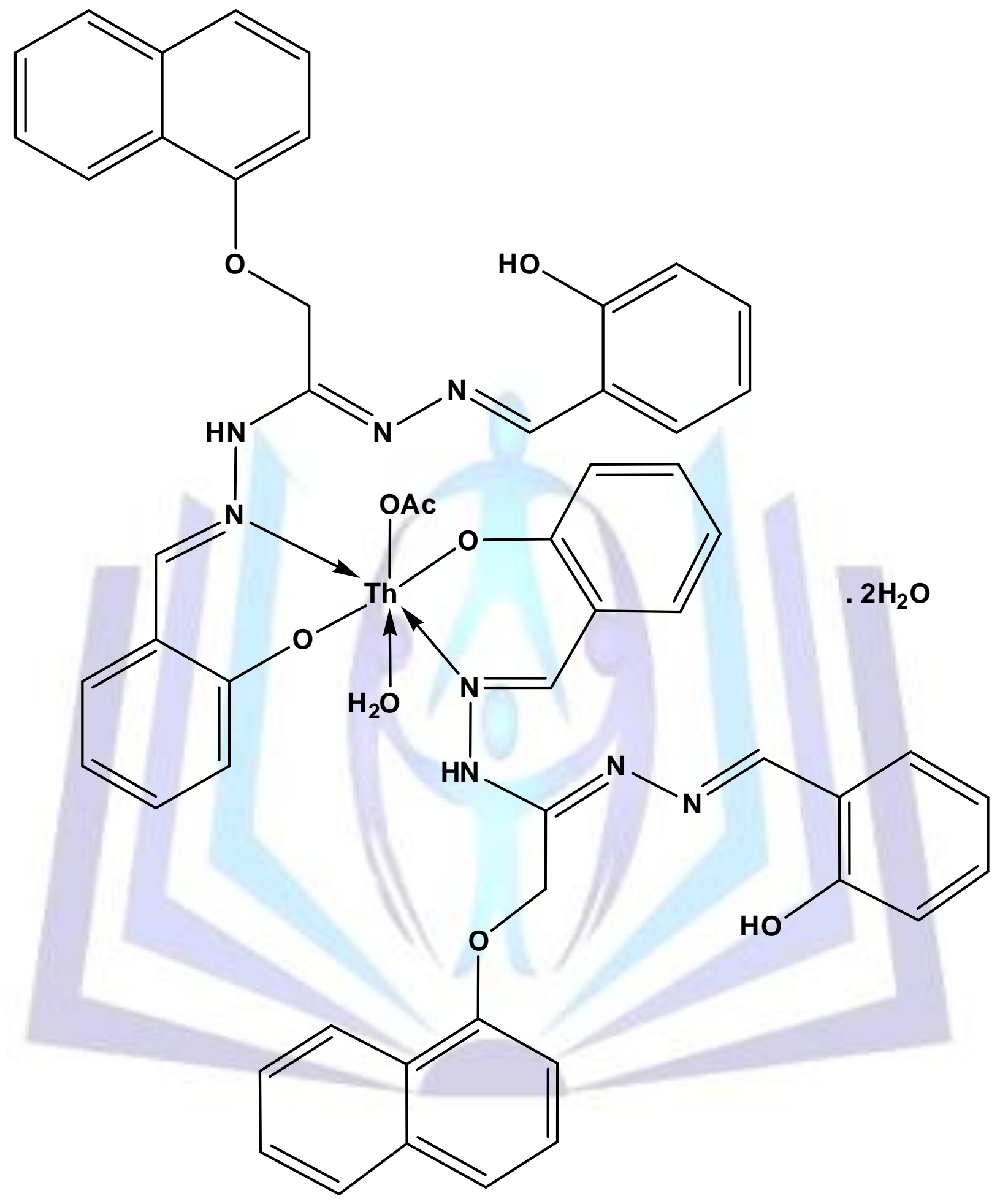

Complex (19)

Figure 2. Proposed structures of the ligand $\left[\mathrm{H}_{2} \mathrm{~L}\right]$ and its metal complexes (2)-(19) 


\section{ISSN 2321-807X}

Table 1:-Analytical and Physical Data of the Ligand $\left[\mathrm{H}_{2} L\right](1)$ and its Metal Complexes.

\begin{tabular}{|c|c|c|c|c|c|c|c|c|c|c|c|}
\hline \multirow{2}{*}{ No. } & \multirow{2}{*}{ Ligands/Complexes } & \multirow{2}{*}{ Color } & \multirow{2}{*}{ FW } & \multirow{2}{*}{$\begin{array}{l}\text { M.P } \\
\left({ }^{\mathrm{o}} \mathrm{C}\right)\end{array}$} & \multirow{2}{*}{$\begin{array}{l}\text { Yield } \\
(\%)\end{array}$} & \multicolumn{4}{|c|}{ Anal. /Found (Calc.) (\%) } & \multirow[b]{2}{*}{$\mathbf{C l}$} & \multirow{2}{*}{$\begin{array}{c}\text { Molar } \\
\text { conductance* }\end{array}$} \\
\hline & & & & & & $\mathbf{C}$ & $\mathbf{H}$ & $\mathbf{N}$ & $\mathbf{M}$ & & \\
\hline (1) & $\begin{array}{l}{\left[\mathrm{H}_{2} \mathrm{~L}\right]} \\
\mathrm{C}_{26} \mathrm{H}_{22} \mathrm{~N}_{4} \mathrm{O}_{3}\end{array}$ & Beige & 438.48 & 195 & 75 & $71.87(71.22)$ & $4.71(5.06)$ & $12.23(12.78)$ & - & - & - \\
\hline (2) & $\begin{array}{l}{\left[(\mathrm{L}) \mathrm{Co}\left(\mathrm{H}_{2} \mathrm{O}\right)_{2}\right] \cdot \mathrm{H}_{2} \mathrm{O}} \\
\mathrm{C}_{26} \mathrm{H}_{26} \mathrm{Co} \mathrm{N}_{4} \mathrm{O}_{6}\end{array}$ & Orange & 549.44 & $>300$ & 80 & $56.67(56.84)$ & $4.75(4.77)$ & $10.371(10.20)$ & $10.61(10.73)$ & - & 6.5 \\
\hline (3) & $\begin{array}{l}{\left[(\mathrm{HL})_{2}(\mathrm{Ni})_{3}\left(\mathrm{SO}_{4}\right)_{2}\left(\mathrm{H}_{2} \mathrm{O}\right)_{8}\right] \cdot 2 \mathrm{H}_{2} \mathrm{O}} \\
\mathrm{C}_{52} \mathrm{H}_{62} \mathrm{~N}_{8} \mathrm{Ni}_{3} \mathrm{O}_{24} \mathrm{~S}_{2}\end{array}$ & Beige & 1423.30 & $>300$ & 84 & $44.18(43.88)$ & $4.0(4.39)$ & $7.9(7.87)$ & $12.23(12.37)$ & - & 14.8 \\
\hline (4) & $\begin{array}{l}{\left[(\mathrm{L}) \mathrm{Ni}\left(\mathrm{H}_{2} \mathrm{O}\right)_{2}\right] \cdot 2 \mathrm{H}_{2} \mathrm{O}} \\
\mathrm{C}_{26} \mathrm{H}_{28} \mathrm{~N}_{4} \mathrm{Ni} \mathrm{O}_{7}\end{array}$ & Olive & 567.22 & $>300$ & 69 & $55.18(55.05)$ & $4.98(4.98)$ & $9.248(9.88)$ & $10.11(10.35)$ & - & 6.3 \\
\hline$(5)$ & $\begin{array}{l}{\left[(\mathrm{L}) \mathrm{Cu}\left(\mathrm{H}_{2} \mathrm{O}\right)_{2}\right] \cdot 2 \mathrm{H}_{2} \mathrm{O}} \\
\mathrm{C}_{26} \mathrm{H}_{28} \mathrm{Cu} \mathrm{N} \mathrm{O}_{4} \mathrm{O}_{7}\end{array}$ & $\begin{array}{l}\text { Dark } \\
\text { green }\end{array}$ & 572.07 & $>300$ & 71 & $54.24(54.59)$ & $4.49(4.93)$ & $9.86(9.79)$ & $10.8(11.11)$ & - & 13.2 \\
\hline$(6)$ & $\begin{array}{l}{\left[\left(\mathrm{H}_{2} \mathrm{~L}\right)(\mathrm{Ag})_{2}\left(\mathrm{NO}_{3}\right)_{2}\right] \cdot \mathrm{H}_{2} \mathrm{O}} \\
\mathrm{C}_{26} \mathrm{H}_{24} \mathrm{Ag}_{2} \mathrm{~N}_{6} \mathrm{O}_{10}\end{array}$ & Beige & 796.24 & 236 & 80 & $38.9(39.22)$ & $2.98(3.04)$ & $10.33(10.55)$ & $26.8(27.09)$ & - & 7.85 \\
\hline (7) & $\begin{array}{l}{\left[(\mathrm{HL})_{2}(\mathrm{Cu})_{3}(\mathrm{Cl})_{4}\right] \cdot \mathrm{H}_{2} \mathrm{O}} \\
\mathrm{C}_{52} \mathrm{H}_{44} \mathrm{Cl}_{4} \mathrm{Cu}_{3} \mathrm{~N}_{8} \mathrm{O}_{7}\end{array}$ & Beige & 1225.41 & 210 & 72 & $50.81(50.97)$ & $3.98(3.62)$ & $9.0(9.14)$ & $15.1(15.56)$ & $11.4(11.57)$ & 17.3 \\
\hline (8) & $\begin{array}{l}{\left[(\mathrm{HL})_{2}(\mathrm{Fe})_{3}\left(\mathrm{SO}_{4}\right)_{2}\left(\mathrm{H}_{2} \mathrm{O}\right)_{8}\right] \cdot \mathrm{H}_{2} \mathrm{O}} \\
\mathrm{C}_{52} \mathrm{H}_{60} \mathrm{Fe}_{3} \mathrm{~N}_{8} \mathrm{O}_{23} \mathrm{~S}_{2}\end{array}$ & Olive & 1396.74 & 242 & 68 & $44.40(44.72)$ & $4.1(4.33)$ & $7.9(8.02)$ & 11.33(11.99) & - & 16.4 \\
\hline (9) & $\begin{array}{l}{\left[(\mathrm{HL})_{2} \mathrm{~Pb}(\mathrm{OAc})_{2}\right] \cdot 4 \mathrm{H}_{2} \mathrm{O}} \\
\mathrm{C}_{56} \mathrm{H}_{58} \mathrm{~N}_{8} \mathrm{O}_{14} \mathrm{~Pb}\end{array}$ & Grey & 1274.30 & 223 & 65 & $52.38(52.78)$ & $4.22(4.59)$ & $8.65(8.79)$ & 16.1(16.26) & - & 11.3 \\
\hline (10) & $\begin{array}{l}{\left[(\mathrm{HL})_{2} \mathrm{Mn}\left(\mathrm{H}_{2} \mathrm{O}\right)_{2} \cdot 4 \mathrm{H}_{2} \mathrm{O}\right.} \\
\mathrm{C}_{52} \mathrm{H}_{54} \mathrm{Mn} \mathrm{N} \mathrm{N}_{8} \mathrm{O}_{12}\end{array}$ & Brown & 1037.97 & 238 & 6 & $59.80(60.17)$ & $5.1(5.24)$ & $10.6(10.80)$ & $4.78(5.29)$ & - & 7.5 \\
\hline (11) & $\begin{array}{l}{\left[(\mathrm{HL})_{2}(\mathrm{Co})_{3}\left(\mathrm{SO}_{4}\right)_{2}\left(\mathrm{H}_{2} \mathrm{O}\right)_{2}\right] \cdot 2 \mathrm{H}_{2} \mathrm{O}} \\
\mathrm{C}_{52} \mathrm{H}_{50} \mathrm{Co}_{3} \mathrm{~N}_{8} \mathrm{O}_{18} \mathrm{~S}_{2}\end{array}$ & Beige & 1315.93 & 234 & 89 & $47.20(47.46)$ & $3.5(3.83)$ & $8.3(8.52)$ & $13.0(13.44)$ & - & 13.8 \\
\hline (12) & $\begin{array}{l}{\left[(\mathrm{HL}) \mathrm{Cu}\left(\mathrm{NO}_{3}\right)\left(\mathrm{H}_{2} \mathrm{O}\right)\right] \cdot 2 \mathrm{H}_{2} \mathrm{O}} \\
\mathrm{C}_{26} \mathrm{H}_{27} \mathrm{Cu} \mathrm{N} \mathrm{N}_{5} \mathrm{O}_{9}\end{array}$ & Brown & 617.07 & 230 & 55 & $50.55(50.61)$ & $4.37(4.41)$ & $11.34(11.35)$ & $10.29(10.30)$ & - & 6.8 \\
\hline (13) & {$\left[(\mathrm{HL})_{2} \mathrm{Co}\left(\mathrm{H}_{2} \mathrm{O}\right)\right] \cdot 2 \mathrm{H}_{2} \mathrm{O}$} & Beige & 1005.93 & 220 & 82 & $62.24(62.09)$ & $4.62(5.01)$ & $11.77(11.14)$ & $6.19(5.86)$ & - & 15.6 \\
\hline
\end{tabular}

3901 | $\mathrm{P}$ a g e 


\section{ISSN 2321-807X}

\begin{tabular}{|c|c|c|c|c|c|c|c|c|c|c|c|}
\hline & $\mathrm{C}_{52} \mathrm{H}_{50} \mathrm{CoN}_{8} \mathrm{O}_{10}$ & & & & & & & & & & \\
\hline (14) & $\begin{array}{l}{\left[(\mathrm{HL})_{2} \mathrm{Cd}(\mathrm{OAc})_{2}\right] .2 \mathrm{H}_{2} \mathrm{O}} \\
\mathrm{C}_{56} \mathrm{H}_{54} \mathrm{Cd} \mathrm{N}_{8} \mathrm{O}_{12}\end{array}$ & Yellow & 1143.49 & $>300$ & 70 & $58.91(58.82)$ & $4.14(4.76)$ & $9.99(9.80)$ & $9.73(9.83)$ & - & 11.2 \\
\hline (15) & $\begin{array}{l}{\left[\left(\mathrm{H}_{2} \mathrm{~L}\right) \mathrm{Zn}(\mathrm{OAc})_{2}\right] \cdot 2 \mathrm{H}_{2} \mathrm{O}} \\
\mathrm{C}_{30} \mathrm{H}_{32} \mathrm{~N}_{4} \mathrm{O}_{9} \mathrm{Zn}\end{array}$ & Yellow & 657.98 & 223 & 74 & $54.34(54.76)$ & 4.01(4.90) & $8.03(8.52)$ & $9.8(9.94)$ & - & 12.1 \\
\hline (16) & $\begin{array}{l}{\left[(\mathrm{HL})_{2}(\mathrm{Cu})_{3}\left(\mathrm{SO}_{4}\right)_{2}\left(\mathrm{H}_{2} \mathrm{O}\right)_{8}\right] \cdot \mathrm{H}_{2} \mathrm{O}} \\
\mathrm{C}_{52} \mathrm{H}_{60} \mathrm{Cu}_{3} \mathrm{~N}_{8} \mathrm{O}_{23} \mathrm{~S}_{2}\end{array}$ & Silver & 1419.84 & 216 & 66 & $43.79(43.99)$ & $4.16(4.26)$ & 7.12(7.89) & $12.98(13.43)$ & - & 13.7 \\
\hline (17) & $\begin{array}{l}{\left[(\mathrm{HL})_{2} \mathrm{Hg}\left(\mathrm{H}_{2} \mathrm{O}\right)_{2}\right] \cdot \mathrm{H}_{2} \mathrm{O}} \\
\mathrm{C}_{52} \mathrm{H}_{48} \mathrm{Hg} \mathrm{N}_{8} \mathrm{O}_{9}\end{array}$ & Beige & 1129.58 & 230 & 73 & $54.8(55.29)$ & $4.0(4.28)$ & $9.7(9.92)$ & 17.3(17.76) & - & 6.8 \\
\hline (18) & $\begin{array}{l}{\left[(\mathrm{HL})_{2} \mathrm{Sr}\left(\mathrm{H}_{2} \mathrm{O}\right)_{2}\right] \cdot 4 \mathrm{H}_{2} \mathrm{O}} \\
\mathrm{C}_{52} \mathrm{H}_{54} \mathrm{~N}_{8} \mathrm{O}_{12} \mathrm{Sr}\end{array}$ & Beige & 1070.65 & 240 & 76 & 58.1(58.33) & $4.8(5.08)$ & $10.2(10.47)$ & $7.89(8.18)$ & - & 7.2 \\
\hline (19) & $\begin{array}{l}{\left[(\mathrm{HL})_{2} \mathrm{Th}(\mathrm{OAc})\left(\mathrm{H}_{2} \mathrm{O}\right)\right] \cdot 2 \mathrm{H}_{2} \mathrm{O}} \\
\mathrm{C}_{54} \mathrm{H}_{51} \mathrm{~N}_{8} \mathrm{O}_{11} \mathrm{Th}\end{array}$ & Beige & 1220.07 & 245 & 60 & $52.89(53.16)$ & $4.1(4.21)$ & $8.9(9.18)$ & 18.77(19.02) & - & 8.3 \\
\hline
\end{tabular}

$* \mathrm{M}\left({ }^{-1} \mathrm{~cm}^{2} \mathrm{~mol}^{-1}\right)$

Table 2:- IR Frequencies of the Bands $\left(\mathrm{cm}^{-1}\right)$ of Ligand $\left[\mathrm{H}_{2} \mathrm{~L}\right]$, (1) and its Metal Complexes

\begin{tabular}{|c|c|c|c|c|c|c|c|c|c|c|c|c|}
\hline No. & $v\left(\mathrm{H}_{2} \mathrm{O}\right)$ & $v(\mathrm{OH})$ & $\begin{array}{c}v(\mathrm{H}- \\
\text { bonding) }\end{array}$ & $v(\mathrm{NH})$ & $v(N-N)$ & $v(C=N)$ & $\mathbf{v}(\mathrm{COH} / \mathrm{CO})$ & $v(A r)$ & $\mathrm{v}(\mathrm{OAc}) / \mathrm{SO} 4 / \mathrm{NO}_{3}$ & v(M-O) & $v(M-N)$ & v(M-Cl) \\
\hline \multirow{2}{*}{ (1) } & \multirow{2}{*}{-} & \multirow{2}{*}{3465,3446} & $3650-3310$ & \multirow{2}{*}{3220} & \multirow{2}{*}{1031} & \multirow{2}{*}{1623,1618} & \multirow{2}{*}{1316} & 1573,784 & & \multirow{2}{*}{ - } & \multirow[b]{2}{*}{-} & \\
\hline & & & $3280-2650$ & & & & & 1550,752 & & & & - \\
\hline \multirow{2}{*}{ (2) } & \multirow{2}{*}{$3550-3480$} & \multirow[b]{2}{*}{ - } & $3620-3280$ & \multirow{2}{*}{3230} & \multirow{2}{*}{1040} & \multirow{2}{*}{1615,1605} & \multirow{2}{*}{1301} & 1541,791 & & \multirow{2}{*}{587} & \multirow{2}{*}{453} & \\
\hline & & & $3270-2850$ & & & & & 1471,757 & & & & - \\
\hline \multirow{2}{*}{ (3) } & \multirow{2}{*}{$3550-3490$} & \multirow{2}{*}{3430} & $3610-3330$ & 3238 & \multirow{2}{*}{1039} & \multirow{2}{*}{$\begin{array}{c}1623,1618 \\
1610\end{array}$} & \multirow{2}{*}{1316,1305} & 1571,783 & 1157,1147 & \multirow{2}{*}{618} & \multirow{2}{*}{565} & \\
\hline & & & $3320-2650$ & 3225 & & & & 1535,751 & 730,682 & & & \\
\hline \multirow{2}{*}{ (4) } & \multirow{2}{*}{$3550-3480$} & \multirow{2}{*}{ - } & $3650-3210$ & \multirow{2}{*}{3220} & \multirow{2}{*}{1037} & \multirow{2}{*}{1619,1600} & \multirow{2}{*}{1309} & 1560,784 & \multirow{2}{*}{-} & \multirow{2}{*}{587} & \multirow{2}{*}{545} & \\
\hline & & & $3200-2670$ & & & & & 1550,754 & & & & - \\
\hline$(5)$ & $3530-3485$ & 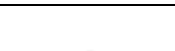 & $3620-3280$ & 3241 & 1035 & 16141605 & 1305 & 1536756 & 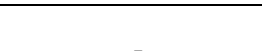 & 500 & 468 & \\
\hline (J) & J & - & $3270-2680$ & 5241 & 1053 & 1014,1005 & 1300 & $1550, / 50$ & - & 590 & 408 & - \\
\hline (6) & $3650-3540$ & 3466,3447 & $3620-3320$ & 3225 & 1037 & 1624,1622 & 1316 & $1572-752$ & 1330,1148 & 565 & 450 & \\
\hline
\end{tabular}

3902 | P a g e

$\mathrm{JuIy} 16,2015$ 
ISSN 2321-807X

\begin{tabular}{|c|c|c|c|c|c|c|c|c|c|c|c|c|}
\hline & & & $3300-2650$ & & & & & & 890,752 & & & - \\
\hline (7) & $3520-3475$ & 3432 & $\begin{array}{l}3602-3310 \\
3300-2720\end{array}$ & 3223 & 1038 & 1619,1610 & 1316,1308 & 1572,748 & - & 575 & 465 & 415 \\
\hline (8) & $3510-3470$ & 3446 & $\begin{array}{l}3580-3210 \\
3200-2750\end{array}$ & 3231 & 1037 & 1623,1618 & 1316,1307 & 1517,751 & $\begin{array}{c}1197,1157 \\
1147 \\
682\end{array}$ & 617 & 565 & - \\
\hline (9) & $3520-3465$ & 3453,3435 & $\begin{array}{l}3570-3280 \\
3270-2760\end{array}$ & 3217 & 1037 & 1624,1616 & 1316 & 1572,784 & 1447,1330 & 565 & 459 & - \\
\hline (10) & $3530-3450$ & 3445 & $\begin{array}{l}3610-3330 \\
3320-2850\end{array}$ & 3322 & 1039 & 1624,1618 & 1316,1307 & 1572,752 & 1455,1330 & 565 & 459 & - \\
\hline (11) & $3500-3465$ & 3430 & $\begin{array}{l}3580-3280 \\
3270-2680\end{array}$ & 3231 & 1036 & 1622 & 1315,1309 & 1572,750 & $\begin{array}{c}1195,1156 \\
1140 \\
681,458\end{array}$ & 618 & 564 & - \\
\hline (12) & $3560-3350$ & 3456 & $\begin{array}{l}3600-3210 \\
3200-2680\end{array}$ & 3225 & 1039 & 1623,1618 & 1316,1307 & 1533,752 & $\begin{array}{c}1327,1128 \\
894,785\end{array}$ & 592 & 565 & - \\
\hline (13) & $3565-3470$ & 3445 & $\begin{array}{l}3610-3315 \\
3310-2650\end{array}$ & 3222 & 1037 & 1624,1618 & 1308 & 1572,783 & - & 546 & 550 & - \\
\hline (14) & $3500-3480$ & 3451,3446 & $\begin{array}{l}3600-3320 \\
3310-2750\end{array}$ & 3226 & 1037 & 1624,1620 & 1317 & 1572,752 & 1435,1335 & 565 & 460 & - \\
\hline (15) & 3560 & 3455,3431 & $\begin{array}{l}3600-3300 \\
3290-2650\end{array}$ & 3235 & 1038 & 1624,1619 & 1315 & 1571,752 & 1471,1341 & 605 & 550 & - \\
\hline (16) & $3550-3470$ & 3437 & $\begin{array}{l}3580-3280 \\
3270-2680\end{array}$ & 3232 & 1037 & 1625,1620 & 1316,1308 & 1572,752 & $\begin{array}{c}1206,1156 \\
1147 \\
682,458\end{array}$ & 618 & 565 & - \\
\hline (17) & $3520-3465$ & 3435 & $\begin{array}{l}3600-3260 \\
3250-2630\end{array}$ & 3220 & 1037 & 1624,1618 & 1316,1308 & 1572,751 & - & 600 & 550 & - \\
\hline (18) & 3520 & 3435 & $\begin{array}{l}3580-3300 \\
3290-2650\end{array}$ & 3222 & 1037 & 1625,1620 & 1316,1307 & 1572,751 & - & 580 & 520 & - \\
\hline (19) & 3510 & 3437 & $\begin{array}{l}3580-3285 \\
3275-2700\end{array}$ & 3325 & 1037 & 1624,1620 & 1316,1307 & 1571,752 & 1446,1330 & 565 & 465 & - \\
\hline
\end{tabular}




\subsection{Conductivity measurements}

The molar conductivity of $110^{-3} \mathrm{M}$ solution of the metal complexes (2-19) in DMSO at room temperature are given in experimental section. The value of molar conductance of all complexes is in the $6.3-16.4^{-1} \mathrm{~cm}^{2} \mathrm{~mol}{ }^{-1}$ range indicating a non-electrolytic nature of these complexes confirming the involvement of the acetate, sulfate, nitrate and chloride anions in the coordination sphere.

\subsection{Mass spectra}

The mass spectrum of the ligand (1) revealed a molecular ion peak $(\mathrm{m} / \mathrm{z})$ at 438 a.m.u which is coincident with the formula weight of the ligand and supports the identity of the Structure. Furthermore, the fragments observed at $\mathrm{m} / \mathrm{z}=30$, 43, 72, 77, 86, 91, 107, 117, 133, 147, 194, 206, 236, 284, 326,398 and 438 corresponding to $\mathrm{C}_{2} \mathrm{H}_{6}, \mathrm{C}_{3} \mathrm{H}_{7}, \mathrm{C}_{5} \mathrm{H}_{12}, \mathrm{C}_{6} \mathrm{H}_{5}$, $\mathrm{C}_{7} \mathrm{H}_{2}, \quad \mathrm{C}_{7} \mathrm{H}_{7}, \mathrm{C}_{8} \mathrm{H}_{11}, \mathrm{C}_{8} \mathrm{H}_{50}, \mathrm{C}_{8} \mathrm{H}_{5} \mathrm{O}_{2}, \mathrm{C}_{9} \mathrm{H}_{7} \mathrm{O}_{2}, \mathrm{C}_{14} \mathrm{H}_{12} \mathrm{~N}, \mathrm{C}_{15} \mathrm{H}_{12} \mathrm{~N}, \mathrm{C}_{16} \mathrm{H}_{14} \mathrm{NO}, \mathrm{C}_{20} \mathrm{H}_{14} \mathrm{NO}, \mathrm{C}_{22} \mathrm{H}_{16} \mathrm{NO}_{2}, \mathrm{C}_{24} \mathrm{H}_{22} \mathrm{~N}_{4} \mathrm{O}_{2}$ and $\mathrm{C}_{26} \mathrm{H}_{22} \mathrm{~N}_{4} \mathrm{O}_{3}$ moieties respectively. However, the spectrum of $\mathrm{Cu}(\mathrm{II})$ complex (5) spectrum shows a peak (m/z) at 571 a.m.u corresponding to the formula weight of the complex. Additionally, the peaks observed at 30,41, 57, 63, 81, 98, 118, 180, 198, 228, 250, 279, 322, 337, 365, 393, 412, 438, 517 and 571 are due to $\mathrm{C}_{2} \mathrm{H} 6, \mathrm{C}_{3} \mathrm{H}_{5}, \mathrm{C}_{4} \mathrm{H}_{9}, \mathrm{C}_{3} \mathrm{H}_{11} \mathrm{O}, \mathrm{C}_{4} \mathrm{H}_{17} \mathrm{O}, \mathrm{C}_{5} \mathrm{H}_{8} \mathrm{NO}$, $\mathrm{C}_{5} \mathrm{H}_{8} \mathrm{NO}, \mathrm{C}_{6} \mathrm{H}_{16} \mathrm{NO}, \mathrm{C}_{9} \mathrm{H}_{12} \mathrm{~N}_{2} \mathrm{O}_{2}, \mathrm{C}_{10} \mathrm{H}_{18} \mathrm{~N}_{2} \mathrm{O}_{2}, \mathrm{C}_{11} \mathrm{H}_{20} \mathrm{~N}_{2} \mathrm{O}_{3}, \mathrm{C}_{14} \mathrm{H}_{22} \mathrm{~N}_{2} \mathrm{O}_{2}, \mathrm{C}_{16} \mathrm{H}_{11} \mathrm{~N}_{2} \mathrm{O}_{3}, \mathrm{C}_{19} \mathrm{H}_{18} \mathrm{~N}_{2} \mathrm{O}_{3}, \mathrm{C}_{20} \mathrm{H}_{21} \mathrm{~N}_{2} \mathrm{O}_{3}, \mathrm{C}_{20} \mathrm{H}_{19} \mathrm{~N}_{3} \mathrm{O}_{4}$ $\mathrm{C}_{21} \mathrm{H}_{19} \mathrm{~N}_{3} \mathrm{O}_{5}, \mathrm{C}_{21} \mathrm{H}_{19} \mathrm{CuNO}_{4}, \mathrm{C}_{22} \mathrm{H}_{19} \mathrm{CuN}_{2} \mathrm{O}_{4}, \mathrm{C}_{24} \mathrm{H}_{28} \mathrm{CuN}_{3} \mathrm{O}_{6}$ and $\mathrm{C}_{26} \mathrm{H}_{28} \mathrm{CuN}_{4} \mathrm{O}_{7}$ moieties respectively. The spectrum of $\mathrm{Zn}$ (II) complex (15) spectrum shows a peak (m/z) at 657 a.m.u corresponding to the formula weight of the complex. Additionally, the peaks observed at 18, 28, 44, 65, 77, 93, 109, 141, 169, 195, 212, 226, 251, 265, 304, 321, 337, 359, 395, 413, 448, 547, 577, 607,639 and 657 are due to $\mathrm{CH}_{6}, \mathrm{C}_{2} \mathrm{H}_{4}, \mathrm{C}_{3} \mathrm{H}_{8}, \mathrm{C}_{5} \mathrm{H}_{5}, \mathrm{C}_{6} \mathrm{H}_{5}, \mathrm{C}_{7} \mathrm{H}_{9}, \mathrm{C}_{8} \mathrm{H}_{13}, \mathrm{C}_{9} \mathrm{H}_{17} \mathrm{O}, \mathrm{C}_{10} \mathrm{H}_{17} \mathrm{O}_{2}$, $\mathrm{C}_{12} \mathrm{H}_{19} \mathrm{O}_{2}, \mathrm{C}_{12} \mathrm{H}_{20} \mathrm{O}_{3}, \mathrm{C}_{12} \mathrm{H}_{20} \mathrm{NO}_{3}, \mathrm{C}_{14} \mathrm{H}_{21} \mathrm{NO}_{3}, \mathrm{C}_{14} \mathrm{H}_{21} \mathrm{~N}_{2} \mathrm{O}_{3}, \mathrm{C}_{17} \mathrm{H}_{24} \mathrm{~N}_{2} \mathrm{O}_{3}, \mathrm{C}_{17} \mathrm{H}_{25} \mathrm{~N}_{2} \mathrm{O}_{4}, \mathrm{C}_{17} \mathrm{H}_{25} \mathrm{~N}_{2} \mathrm{O}_{5}, \mathrm{C}_{25} \mathrm{H}_{15} \mathrm{~N}_{2} \mathrm{O}, \mathrm{C}_{28} \mathrm{H}_{15} \mathrm{~N}_{2} \mathrm{O}$, $\mathrm{C}_{28} \mathrm{H}_{17} \mathrm{~N}_{2} \mathrm{O}_{2}, \mathrm{C}_{28} \mathrm{H}_{20} \mathrm{~N}_{2} \mathrm{O}_{4}, \mathrm{C}_{28} \mathrm{H}_{24} \mathrm{~N}_{3} \mathrm{O} \mathrm{Zn}, \mathrm{C}_{29} \mathrm{H}_{26} \mathrm{~N}_{3} \mathrm{O}_{6} \mathrm{Zn}, \mathrm{C}_{30} \mathrm{H}_{28} \mathrm{~N}_{3} \mathrm{O}_{7} \mathrm{Zn}, \mathrm{C}_{30} \mathrm{H}_{28} \mathrm{~N}_{4} \mathrm{O}_{8} \mathrm{Zn}$ and $\mathrm{C}_{30} \mathrm{H}_{30} \mathrm{~N}_{4} \mathrm{O}_{9} \mathrm{Zn}$ moieties respectively. The fragments of the ligand (1), Cu(II) complex (5) and $\mathrm{Zn}(\mathrm{II})$ complex (15) are represented in Table 3.

Table 3: Mass spectra of $\left[\mathrm{H}_{2} \mathrm{~L}\right](1), \mathrm{Cu}(\mathrm{II})$ complex (5) and $\mathrm{Zn}(\mathrm{II})$ complex (15) i. Mass spectrum of the ligand [ $\left.\mathrm{H}_{2} \mathrm{~L}\right]$

\begin{tabular}{|c|c|c|}
\hline $\mathbf{m} / \mathbf{z}$ & Rel. In & Assignments \\
\hline 30 & 49 & $\left(\mathrm{C}_{2} \mathrm{H}_{6}\right)$ \\
\hline 43 & 14 & $\left(\mathrm{C}_{3} \mathrm{H}_{7}\right)$ \\
\hline 72 & 37 & $\left(\mathrm{C}_{5} \mathrm{H}_{12}\right)$ \\
\hline 77 & 6 & $\left(\mathrm{C}_{6} \mathrm{H}_{5}\right)$ \\
\hline 86 & 8 & $\left(\mathrm{C}_{7} \mathrm{H}_{2}\right)$ \\
\hline 91 & 13 & $\left(\mathrm{C}_{7} \mathrm{H}_{7}\right)$ \\
\hline 107 & 25 & $\left(\mathrm{C}_{8} \mathrm{H}_{11}\right)$ \\
\hline 117 & 100 & $\left(\mathrm{C}_{8} \mathrm{H}_{5} \mathrm{O}\right)$ \\
\hline 133 & 38 & $\left(\mathrm{C}_{8} \mathrm{H}_{5} \mathrm{O}_{2}\right)$ \\
\hline 147 & 55 & $\left(\mathrm{C}_{9} \mathrm{H}_{7} \mathrm{O}_{2}\right)$ \\
\hline 194 & 30 & $\left(\mathrm{C}_{14} \mathrm{H}_{12} \mathrm{~N}\right)$ \\
\hline 206 & 2 & $\left(\mathrm{C}_{15} \mathrm{H}_{12} \mathrm{~N}\right)$ \\
\hline 236 & 13 & $\left(\mathrm{C}_{16} \mathrm{H}_{14} \mathrm{NO}\right)$ \\
\hline 284 & 5 & $\left(\mathrm{C}_{20} \mathrm{H}_{14} \mathrm{NO}\right)$ \\
\hline 326 & 12 & $\left(\mathrm{C}_{22} \mathrm{H}_{16} \mathrm{NO}_{2}\right)$ \\
\hline 398 & 8 & $\left(\mathrm{C}_{24} \mathrm{H}_{22} \mathrm{~N}_{4} \mathrm{O}_{2}\right)$ \\
\hline 438 & 5 & $\left(\mathrm{C}_{26} \mathrm{H}_{22} \mathrm{~N}_{4} \mathrm{O}_{3}\right)$ \\
\hline
\end{tabular}


ii. Mass spectrum of $\mathrm{Cu}$ (II) complex (5)

\begin{tabular}{|c|c|c|}
\hline $\mathbf{m} / \mathbf{z}$ & Rel. Int. & Fragment \\
\hline 30 & 39 & $\left(\mathrm{C}_{2} \mathrm{H}_{6}\right)$ \\
\hline 41 & 24 & $\left(\mathrm{C}_{3} \mathrm{H}_{5}\right)$ \\
\hline 57 & 27 & $\left(\mathrm{C}_{4} \mathrm{H}_{9}\right)$ \\
\hline 63 & 11 & $\left(\mathrm{C}_{3} \mathrm{H}_{11} \mathrm{O}\right)$ \\
\hline 81 & 11 & $\left(\mathrm{C}_{4} \mathrm{H}_{17} \mathrm{O}\right)$ \\
\hline 98 & 12 & $\left(\mathrm{C}_{5} \mathrm{H}_{8} \mathrm{NO}\right)$ \\
\hline 118 & 10 & $\left(\mathrm{C}_{6} \mathrm{H}_{16} \mathrm{NO}\right)$ \\
\hline 180 & 6 & $\left(\mathrm{C}_{9} \mathrm{H}_{12} \mathrm{~N}_{2} \mathrm{O}_{2}\right)$ \\
\hline 198 & 8 & $\left(\mathrm{C}_{10} \mathrm{H}_{18} \mathrm{~N}_{2} \mathrm{O}_{2}\right)$ \\
\hline 228 & 12 & $\left(\mathrm{C}_{11} \mathrm{H}_{20} \mathrm{~N}_{2} \mathrm{O}_{3}\right)$ \\
\hline 250 & 25 & $\left(\mathrm{C}_{14} \mathrm{H}_{22} \mathrm{~N}_{2} \mathrm{O}_{2}\right)$ \\
\hline 279 & 8 & $\left(\mathrm{C}_{16} \mathrm{H}_{11} \mathrm{~N}_{2} \mathrm{O}_{3}\right)$ \\
\hline 322 & 100 & $\left(\mathrm{C}_{19} \mathrm{H}_{18} \mathrm{~N}_{2} \mathrm{O}_{3}\right)$ \\
\hline 337 & 11 & $\left(\mathrm{C}_{20} \mathrm{H}_{21} \mathrm{~N}_{2} \mathrm{O}_{3}\right)$ \\
\hline 365 & 13 & $\left(\mathrm{C}_{20} \mathrm{H}_{19} \mathrm{~N}_{3} \mathrm{O}_{4}\right)$ \\
\hline 393 & 26 & $\left(\mathrm{C}_{21} \mathrm{H}_{19} \mathrm{~N}_{3} \mathrm{O}_{5}\right)$ \\
\hline 412 & 10 & $\left(\mathrm{C}_{21} \mathrm{H}_{19} \mathrm{CuNO}_{4}\right)$ \\
\hline 438 & 9 & $\left(\mathrm{C}_{22} \mathrm{H}_{19} \mathrm{CuN}_{2} \mathrm{O}_{4}\right)$ \\
\hline 517 & 5 & $\left(\mathrm{C}_{24} \mathrm{H}_{28} \mathrm{CuN}_{3} \mathrm{O}_{6}\right)$ \\
\hline 571 & 12 & $\left(\mathrm{C}_{26} \mathrm{H}_{28} \mathrm{CuN}_{4} \mathrm{O}_{7}\right)$ \\
\hline
\end{tabular}

ii. Mass spectrum of $\mathrm{Zn}$ (II) complex (15)

\begin{tabular}{|c|c|c|}
\hline $\mathbf{m} / \mathbf{z}$ & Rel. Int. & Fragment \\
\hline 18 & 37 & $\left(\mathrm{CH}_{6}\right)$ \\
\hline 28 & 90 & $\left(\mathrm{C}_{2} \mathrm{H}_{4}\right)$ \\
44 & 13 & $\left(\mathrm{C}_{3} \mathrm{H}_{8}\right)$ \\
65 & 8 & $\left(\mathrm{C}_{5} \mathrm{H}_{5}\right)$ \\
\hline 77 & 9 & $\left(\mathrm{C}_{6} \mathrm{H}_{5}\right)$ \\
\hline 93 & 7 & $\left(\mathrm{C}_{7} \mathrm{H}_{9}\right)$ \\
\hline 109 & 15 & $\left(\mathrm{C}_{8} \mathrm{H}_{13}\right)$ \\
\hline 141 & 11 & $\left(\mathrm{C}_{9} \mathrm{H}_{17} \mathrm{O}\right)$ \\
\hline 169 & 10 & $\left(\mathrm{C}_{10} \mathrm{H}_{17} \mathrm{O}_{2}\right)$ \\
\hline 195 & 62 & $\left(\mathrm{C}_{12} \mathrm{H}_{19} \mathrm{O}_{2}\right)$ \\
\hline
\end{tabular}




\section{ISSN 2321-807X}

\begin{tabular}{|c|c|c|}
\hline 212 & 92 & $\left(\mathrm{C}_{12} \mathrm{H}_{20} \mathrm{O}_{3}\right)$ \\
\hline 226 & 6 & $\left(\mathrm{C}_{12} \mathrm{H}_{20} \mathrm{NO}_{3}\right)$ \\
\hline 251 & 36 & $\left(\mathrm{C}_{14} \mathrm{H}_{21} \mathrm{NO}_{3}\right)$ \\
\hline 265 & 12 & $\left(\mathrm{C}_{14} \mathrm{H}_{21} \mathrm{~N}_{2} \mathrm{O}_{3}\right)$ \\
\hline 304 & 14 & $\left(\mathrm{C}_{17} \mathrm{H}_{24} \mathrm{~N}_{2} \mathrm{O}_{3}\right)$ \\
\hline 321 & 15 & $\left(\mathrm{C}_{17} \mathrm{H}_{25} \mathrm{~N}_{2} \mathrm{O}_{4}\right)$ \\
\hline 337 & 13 & $\left(\mathrm{C}_{17} \mathrm{H}_{25} \mathrm{~N}_{2} \mathrm{O}_{5}\right)$ \\
\hline 359 & 25 & $\left(\mathrm{C}_{25} \mathrm{H}_{15} \mathrm{~N}_{2} \mathrm{O}\right)$ \\
\hline 395 & 100 & $\left(\mathrm{C}_{28} \mathrm{H}_{15} \mathrm{~N}_{2} \mathrm{O}\right)$ \\
\hline 413 & 14 & $\left(\mathrm{C}_{28} \mathrm{H}_{17} \mathrm{~N}_{2} \mathrm{O}_{2}\right)$ \\
\hline 448 & 15 & $\left(\mathrm{C}_{28} \mathrm{H}_{20} \mathrm{~N}_{2} \mathrm{O}_{4}\right)$ \\
\hline 547 & 12 & $\left(\mathrm{C}_{28} \mathrm{H}_{24} \mathrm{~N}_{3} \mathrm{O}_{5} \mathrm{Zn}\right)$ \\
\hline 577 & 13 & $\left(\mathrm{C}_{29} \mathrm{H}_{26} \mathrm{~N}_{3} \mathrm{O}_{6} \mathrm{Zn}\right)$ \\
\hline 607 & 40 & $\left(\mathrm{C}_{30} \mathrm{H}_{28} \mathrm{~N}_{3} \mathrm{O}_{7} \mathrm{Zn}\right)$ \\
\hline 639 & 20 & $\left(\mathrm{C}_{30} \mathrm{H}_{30} \mathrm{~N}_{4} \mathrm{O}_{8} \mathrm{Zn}\right)$ \\
\hline 657 & 14 & $\left(\mathrm{C}_{30} \mathrm{H}_{32} \mathrm{~N}_{4} \mathrm{O}_{9} \mathrm{Zn}\right)$ \\
\hline
\end{tabular}

\section{3. ${ }^{1} \mathrm{H}-\mathrm{NMR}$ spectra}

The ${ }^{1} \mathrm{H}-\mathrm{NMR}$ spectra of the ligand $\left[\mathrm{H}_{2} \mathrm{~L}\right](1), \mathrm{Cd}(\mathrm{II})$ complex(14) and $\mathrm{Zn}(\mathrm{II})$ complex (15) in deuterated DMSO recorded signals consistent with the proposed structures (Figure 2). The ligand showed a three singlet peaks at 11.2 [2H], $9.0[\mathrm{H}]$ and $8.35[2 \mathrm{H}]$ ppm corresponding to the two protons of the $\mathrm{OH}$, one proton of $\mathrm{NH}$ and two protons of $\mathrm{N}=\mathrm{CH}$ groups respectively [37-39]. The multiplet peaks observed in the 6.94- $7.71 \mathrm{ppm}$ range are assigned to the aromatic protons [11], whereas the singlet signal observed at $2.5 \mathrm{ppm}$, is due to the two protons of the $\mathrm{CH}_{2}$ group [40,41]. However, The spectrum of $\mathrm{Cd}(\mathrm{II})$ complex (14) showed two singlet peaks at 11.2 and $11.0 \mathrm{ppm}$ assigned to non-coordinated and coordinated protons of $\mathrm{OH}$ groups respectively. The singlet peak observed at $8.5 \mathrm{ppm}$ was assigned to the two protons of $\mathrm{NH}$ groups, whereas the multiplet peaks appeared in the 6.94- $7.71 \mathrm{ppm}$ range could be assigned to the aromatic protons. The two singlet signals observed at 8.31 and $2.51 \mathrm{ppm}$ were assigned to $\mathrm{N}=\mathrm{CH}$ and $\mathrm{CH}_{2}$ groups respectively with intensities corresponding to four protons, whereas the two singlet signals corresponding to the six protons of the acetate groups were observed as singlet peaks at $1.9 \mathrm{ppm}$ and $2.1 \mathrm{ppm}$ [42-44]. Spectrum of Zn(II) complex (15) showed a singlet signal at $11.1 \mathrm{ppm}$ due to two protons of the $\mathrm{OH}$ groups; another signal was observed at 9.0 ppm corresponding to one proton of the $\mathrm{NH}$ group. However the aromatic signals was observed in the 6.5-7.71 ppm range. The azomethine $(\mathrm{CH}=\mathrm{N})$ protons were observed as a singlet peaks at 8.49 and $8.40 \mathrm{ppm}$ whereas the signal observed at 2.49 ppm was assigned to protons of $\mathrm{CH}_{2}$ group. The signals observed as two singlet peaks at 1.98 and $2.02 \mathrm{ppm}$ ascribed to the six protons of acetate groups [42-44].

\subsection{Infrared Spectra}

Important spectral bands of the ligand and its complexes are presented in table 2 . The IR spectrum of the ligand showed broad, medium intensity bands in the $3650-3310$ and $3280-2650 \mathrm{~cm}^{-1}$ ranges, which are attributed to intra- and intermolecular hydrogen bondings $[45,46]$. The broad medium bands at 3465 and $3436 \mathrm{~cm}^{-1}$ are assigned to the $(\mathrm{OH}) \mathrm{group}$, whereas the relatively strong bands located at $3220,1623,1618$ and $1316 \mathrm{~cm}^{-1}$, are assigned to the $v(\mathrm{NH})$, phenolic $v(\mathrm{C}=\mathrm{N}), v(\mathrm{C}=\mathrm{N})$ and $v(\mathrm{COH})$ vibrations respectively [47]. Also, the spectrum showed a band at $1031 \mathrm{~cm}^{-1}$ which is assigned to $v(\mathrm{~N}-\mathrm{N})$ vibration $[48,49]$. In order to study the binding mode of the Schiff base to the metal ion in the complexes, the IR spectrum of the free Schiff base was compared with the spectra of the metal complexes. The spectral data together with the elemental analyses indicated that, the ligand can behave as:Bibasic tetradentate ligand: coordinating through the two $\mathrm{O}$ and the two $\mathrm{C}=\mathrm{N}$ groups as in complexes (2), (4), and (5). This mode of coordination is supported by the evidences: (i) the disappearance of the band for the phenolic $\mathrm{OH}$, indicating the subsequent deprotonation of the phenolic proton prior to coordination [50]. (ii) The strong bands observed for the free Schiff base around 1623 and $1618 \mathrm{~cm}^{-1}$, characteristic of the azomethine $(\mathrm{C}=\mathrm{N})$ stretching vibrations were shifted to lower wave numbers, suggesting coordination of the azomethine nitrogen atoms to the metal ion [51,52] (iii)The red shift of the phenolic $\mathrm{CO}$ vibration band toward lower wave number indicating that, the coordination also takes place through the deprotonated phenolic groups [53,54]. (iii) The appearance of new bands in the 453-565 and 587-590 cm regions which are assigned to $\mathrm{u}(\mathrm{M}-\mathrm{N})$ and $\mathrm{u}(\mathrm{M}-\mathrm{O})$ vibrations respectively [55]. Monobasic tetradentate ligand: In complexes (3), (7), (8), (11), (12) 
and (16), each of the two moieties of the ligand participating in the metal complexes coordinating through one $\mathrm{O}^{-}$, two $\mathrm{C}=\mathrm{N}$ and one $\mathrm{OH}$ group, this mode of coordination was supported by the evidences:(i) One vibration band of the two $\mathrm{C}=\mathrm{N}$ was shifted to lower wave number with a decreasing in its intensity while the other one band appeared in its original place [51,52]. (ii) One of the two $\mathrm{OH}$ vibrations bands disappeared in the time that the other one shifted to lower wave number with decreasing its intensity [50]. This indicates that, only one atom of each phenolic oxygens and azomethine nitrogens was involved in the metal coordination. (iii) One band of the two C-O bands was shifted to a higher wave number, while the other was found almost at its original place, indicating that, only one phenolic oxygen was involved in the coordination $[53,54,56]$.(iv) The appearance of new bands in the 564-565 and 575-618 $\mathrm{cm}^{-1}$ regions are due to the $\mathrm{u}(\mathrm{M}-\mathrm{N})$ and $\mathrm{u}(\mathrm{M}-\mathrm{O})$ vibrations respectively [55]. Neutral bidentate ligand: coordinating through one $\mathrm{OH}$ and one $\mathrm{C}=\mathrm{N}$ group as in complexes (6), (9) and (14). This mode of coordination is supported by the following evidences: (i) One band of each of $\mathrm{OH}$ and $\mathrm{C}=\mathrm{N}$ group was shifted to a lower wave number with a decreasing its intensity, while the other ones are found almost at their original place, indicating that, only one of each pair were involved in the coordination [50]. (ii) One band of the two C-O bands was shifted to a higher wave number while the other is found almost at its original place, indicating that, only one phenolic oxygen was involved in the coordination [56]. (iii) The appearance of new bands in the 450-550 and 546-565 $\mathrm{cm}^{-1}$ regions are due to the $\mathrm{U}(\mathrm{M}-\mathrm{N})$ and $\mathrm{u}(\mathrm{M}-\mathrm{O})$ vibrations respectively [55]. Monobasic bidentate ligand: coordinating through one $\mathrm{O}^{-}$and one $\mathrm{C}=\mathrm{N}$ groups as in complexes (10), (13), (17), (18), and (19). This mode of coordination is supported by (i) One of two OH vibrations bands disappeared in the time that the other one appeared at its original place [50].(ii) One vibration band of the two $\mathrm{C}=\mathrm{N}$ was shifted to lower wave number with a decreasing its intensity while the other one band appeared in its original place. (iii) One band of the two $\mathrm{C}-\mathrm{O}$ bands was shifted to a higher wave number while the other is found almost at its original place, indicating that, only one phenolic oxygen was involved in the coordination $[53,54]$. (iv) The appearance of new bands in the $459-550$ and $546-600 \mathrm{~cm}^{-1}$ regions are due to the $\mathrm{u}(\mathrm{M}-\mathrm{N})$ and $\mathrm{u}(\mathrm{M}-$ O) vibrations respectively [55]. Neutral tetradentate ligand: coordinating through two $\mathrm{OH}$ and two $\mathrm{C}=\mathrm{N}$ groups as in complex (15). This mode of coordination is supported by (i) The two vibration bands of each of $\mathrm{OH}$ and $\mathrm{C}=\mathrm{N}$ were shifted to lower wave number with a decreasing in their intensities [50]. (ii) The two bands C-O vibrations were shifted to a higher wave number, indicating participation of the two phenolic oxygen in the metal coordination. (iv) The appearance of new bands in the $420-550$ and 455-605 $\mathrm{cm}^{-1}$ regions corresponding to the $\mathrm{U}(\mathrm{M}-\mathrm{N})$ and $\mathrm{U}(\mathrm{M}-\mathrm{O})$ vibrations respectively [55]. The presence of water molecules within the coordination sphere in all complexes except (6), (9), (14) and (15) were supported by the presence of weak bands around $3560-3380 \mathrm{~cm}^{-1}, 1600-1595 \mathrm{~cm}^{-1}, 895-943 \mathrm{~cm}^{-1}$ and $645-665 \mathrm{~cm}^{-1}$ due to $\mathrm{OH}$ stretching, $\mathrm{HOH}$ deformation, $\mathrm{H}_{2} \mathrm{O}$ rocking and $\mathrm{H}_{2} \mathrm{O}$ wagging, respectively $[57,58]$. The appearance of two characteristic bands in the $1471-1435$ and $1341-1330 \mathrm{~cm}^{-1}$ ranges in the spectra of complexes (9), (14), (15) and (19) were attributed to asym.(COO) and sym.(COO) respectively, indicating the participation of the acetate oxygen in the complex formation [59]. The coordination modes of the acetate group in the complexes were determined by IR spectra, by comparing the separations between the asym. (COO') and sym.(COO-). The

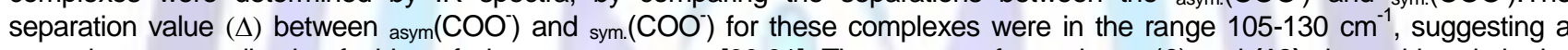
monodentate coordination fashion of the acetate groups [60,61]. The spectra of complexes (6) and (12) showed bands in the $1330-1327,1148-1128,894-890$ and $785-752 \mathrm{~cm}^{-1}$ ranges corresponding to coordinating nitrate group in a unidentate mode [62-64]. Complexes (11) and (16) spectra demonstrated strong to medium bands at 1206, 1195, 1156, 1147, 1140, 682, 681 and $458 \mathrm{~cm}^{-1}$ belonging to the antisymmetric and symmetric stretching modes of the sulfate group. These values are consistent with that reported for the sulfate species coordinating to the $M(I I)$ in an unidentate fashion $[64,38]$. Complexes (7) showed additional band at $415 \mathrm{~cm}^{-1}$ assigned to a coordinated chloride atom.

\subsection{Electronic spectra and magnetic moments.}

DMF electronic absorption spectral bands as well as, room temperature effective magnetic moment values of the ligand and its metal complexes are reported in table 3 . The ligand showed three transition bands in the high energy region. The first band appeared at $290 \mathrm{~nm}$ is assigned to * transition within the aromatic rings and this band is nearly unchanged upon complexation. The second and third bands appearing at 315 and $350 \mathrm{~nm}$ may be assigned to $\mathrm{n}^{*}$ of the azomethine groups and CT transitions $[65,66]$.The bands were found to be shifted upon complexation indicating involvement of theses transition in the coordination with the metal ions. The electronic spectra of the Co(II) complex (2) and (13) exhibit three $d-d$ transition bands at 720, 715; 610, 620, and 560, 550. These bands are assigned to ${ }^{4} T_{1 g}(F) \rightarrow{ }^{4} T_{2 g}(F)\left({ }_{1}\right),{ }^{4} T_{1 g}(F) \rightarrow{ }^{4} T_{1 g}(p)(2),{ }^{4} T_{1 g}(F) \rightarrow{ }^{4} A_{1}(F)\left({ }_{3}\right)$ transitions respectively, corresponding to high spin cobalt(II) octahedral complexes [66,67]. The magnetic moment of complex (2) is 4.98, and 4.74 B.M B.M., which is well within the reported range of high spin octahedral Co(II) complexes. Electronic spectra of $\mathrm{Co}(\mathrm{II})$ complex (11) show bands at 570 and $610 \mathrm{~nm}$. These bands are assigned to ${ }^{4} A_{2 g}(F) \rightarrow{ }^{4} T_{2 g}(P)\left({ }_{3}\right)$ and ${ }^{4} \mathrm{~A}_{2 g}(\mathrm{~F}) \rightarrow{ }^{4} \mathrm{~T}_{1}(\mathrm{~F})(2)$ transitions respectively corresponding to cobalt(II) tetrahedral complexes. The value of the room temperature magnetic moments of complexes (11) is 3.88 B.M., the decrease in the observed magnetic moment (3.88 B.M.) is assigned to spin-spin interactions taking place between $\mathrm{Co}$ (II) ions. The electronic absorption spectra of Ni(II) complexes (3) and (4) displayed three bands at $725-740$ and $608-615 \mathrm{~nm}$, these bands are corresponding to ${ }^{3} \mathrm{~A}_{2 \mathrm{~g}}(\mathrm{~F}) \rightarrow{ }^{3} \mathrm{~T}_{2 \mathrm{~g}}(\mathrm{~F})\left({ }_{1}\right),{ }^{3} \mathrm{~A}_{2 \mathrm{~g}}(\mathrm{~F}) \rightarrow{ }^{3} \mathrm{~T}_{1 \mathrm{~g}}(\mathrm{~F})(2)$ and $\left.{ }^{3} \mathrm{~A}_{2 g}(\mathrm{~F}) \rightarrow{ }^{3} \mathrm{~T}_{1 \mathrm{~g}}(\mathrm{P}) \mathrm{(}_{3}\right)$ transitions respectively, indicating octahedral nickel(II) complexes $[68,69]$. The lower value of $2 / 1$ ratio for the complexes (1.20-1.21) range which are less than the usual range of 1.5-1.75, indicating distorted octahedral nickel(II) complexes $[68,69]$. The magnetic moment values of for nickel(II) complexes (3) and (4) are 2.15 and 3.05 BM respectively, which are consistent with two unpaired electrons state and confirming octahedral geometry for around the nickel(II) ion [68]. The electronic spectra of copper(II) complexes (5), (7), (12) and (16) exhibited bands in the 605-620 and 575-590 nm ranges which are assigned to ${ }^{2} \mathrm{~B}_{1 \mathrm{~g}}{ }^{2} \mathrm{~A}_{1 \mathrm{~g}}\left(\mathrm{~d}_{\mathrm{x}-\mathrm{y}}^{2} \rightarrow \mathrm{d}_{\mathrm{z}}{ }^{2}\right)$, and ${ }^{2} \mathrm{~B}_{1 \mathrm{~g}}{ }^{2} \mathrm{E}_{\mathrm{g}}\left(\mathrm{d}_{\mathrm{x}-\mathrm{y}}{ }^{2} \rightarrow \mathrm{d}_{\mathrm{xy}}, \mathrm{d}_{\mathrm{yz}}\right)$ transitions respectively. These transitions indicate that, the copper(II) ion has a tetragonally distorted octahedral geometry. This could be due to the Jahn-Teller effect that operates on the $\mathrm{d}^{9}$ electronic ground state of six coordinate system, elongating one trans pair of coordinate bonds and shortening the remaining four ones [40, 43]. The electronic spectrum of complex (7) showed peaks at 575 and $620 \mathrm{~nm}$. These bands are assigned to ${ }^{2} B_{1} g^{2} B_{2} g$ and ${ }^{2} B_{1} g^{2} A_{1} g$ transitions, indicating a square planar copper(II) complexes [55,70]. The magnetic moments for all copper(II) complexes at room temperature are in the 1.66-1.74 B.M. range, indicating that, the complexes have octahedral or square planar geometry [71]. The apparent lower values of complexes (12) and (16) may be assigned to spin-spin interactions 
take place between copper(II) ions through molecular interactions[71]. The absorption spectrum of manganese(II) complex (10) showed bands at 585 and $610 \mathrm{~nm}$. These two bands can be assigned to ${ }^{5} \mathrm{~B}_{1 \mathrm{~g}}{ }^{5} \mathrm{E}_{\mathrm{g}}$ and ${ }^{6} \mathrm{~B}_{1 \mathrm{~g}}{ }^{6} \mathrm{~A}_{2 \mathrm{~g}}$ transitions respectively, suggesting an distorted octahedral arrangement around the manganese(II) ion [72,73]. The magnetic moment value for the complex (10) is 5.08 B.M., which is consistent with a high spin octahedral geometry around the manganese(II) ion [74,72]. Diamagnetic cadmium(II), zinc(II), mercury(II), strontium(II), thallium(II) and silver(I) complexes showed only intraligand transitions and (LMCT) (Table 4).

Table 4:- Electronic Spectra (nm) and Magnetic Moments (B.M) for the Ligand,(1) and its metal Complexes.

\begin{tabular}{|c|c|c|c|}
\hline Comp. No. & $\left(\lambda_{\max }(\mathrm{nm}\right.$ & $(\mathrm{BM})_{\mathrm{eff}}$ & $1 / 2$ \\
\hline (1) & $290,315,350$ & - & - \\
\hline (2) & $290,310,330,450,560,610,720$ & 4.98 & 1.18 \\
\hline (3) & $285,307,328,465,575,608,735$ & 2.15 & 1.21 \\
\hline (4) & $285,305,325,495,550,615,740$ & 3.05 & 1.2 \\
\hline (5) & $285,310,336,465,585,615$ & 1.74 & - \\
\hline (6) & $285,295,325,365$ & Diamagnetic & - \\
\hline (7) & $290,315,327,435,575,620$ & 1.63 & - \\
\hline (8) & $285,305,325,406,590,625$ & Diamagnetic & - \\
\hline (9) & $300,326,285$ & Diamagnetic & - \\
\hline (10) & $285,310,325,490,585,610$ & 5.08 & - \\
\hline (11) & $480,570,610,285,300,335$ & 3.88 & - \\
\hline (12) & $290,310,330,465,590,605$ & 1.65 & - \\
\hline (13) & $550,620,715,290,310,335,490$ & 4.74 & - \\
\hline (14) & $335,285,305$ & Diamagnetic & - \\
\hline (15) & $285,305,330$ & Diamagnetic & - \\
\hline (16) & $465,590,610,285,310,325$ & 1.66 & - \\
\hline (17) & $340,285,305$ & Diamagnetic & - \\
\hline (18) & $305,335,285$ & Diamagnetic & - \\
\hline (19) & $305,335,410,285$ & 2.03 & - \\
\hline
\end{tabular}

\subsection{Electron spin resonance (ESR)}

The ESR spectral data for metal complexes (2), (3), (5), (7), (8), (10), (11), (12), (13) and (16) are presented in table 5. Complex (3) showed broad signal in the low and high field regions Indicating spin-exchange interactions take place between $\mathrm{Ni}(\mathrm{II})$ ions which is confirmed by the magnetic moment value. The spectra of copper(II) metal complexes (5), (7), (12) and (16) are characteristic of species, $d^{9}$ configuration and having axial type of a $d\left(x^{2}-y^{2}\right)$ ground state which is the most common for copper(II) complexes [75,76]. The metal complexes showed $\mathrm{g} \|>>2.0023$, indicating octahedral geometry around the copper(II) ion [77]. The expression $\mathrm{G}$ is related to $\mathrm{g}$-values, $\mathrm{G}=\left(\mathrm{g}_{\|}-2\right) /\left(\mathrm{g}_{\perp-2}\right)$. If $\mathrm{G}>4.0$, then local tetragonal axes are misaligned parallel or only slightly misaligned and if $G<4.0$, significant exchange coupling is present [78]. Metal complexes (12) and (16) showed values indicating spin-exchange interactions take place between the copper(II) ions, which is consistent with the of magnetic moments values (Table 4). Also, the $\mathrm{g}\|/ \mathrm{A}\|$ values are considered as a diagnostic of stereochemistry. The $\mathrm{g} \mid / \mathrm{A} \|$ values lie just within the range expected for the octahedral metal complexes [79]. The orbital reduction factors (K\|, K, K), which are a measure of covalencywere also calculated [80]. K values, for the copper(II) complexes (5), (7), (12) and (16), indicating covalent bond character [80]. Also, the g-values show considerable a covalent bond character. The in-plane $\sigma$ - covalency parameter, $\alpha^{2}(\mathrm{Cu})$ suggests a covalent bonding. The complexes show $\beta_{1}^{2}$ values indicating a covalency character in the in-plane $\pi$ - bonding. While $\beta^{2}$ for the complexes indicating a covalent bonding character in the out of plane $\pi$ - bonding except complexes (12) and (16) which indicate ionic bond character $[81,78]$. The calculated orbital populations $\left(a^{2} d\right)$ for the copper(II) complexes indicate a $d\left(x^{2}\right.$ $\mathrm{y}^{2}$ ) ground state [82]. Cobalt(II) complexes (2), (11) and (13), Iron(II) complex (8) and manganese(II) complex (10), show isotropic spectra. 


\subsection{Thermal analyses (DTA and TGA)}

The thermal data of metal complexes (3), (4), (8), (12), (13), (15) and (16) were presented in table 6. The thermal curves in the $27-800^{\circ} \mathrm{C}$ temperature range indicated that the metal complexes are thermally stable up to $40^{\circ} \mathrm{C}$. The weight losses recorded in the $70-90^{\circ} \mathrm{C}$ range is due to elimination of hydrated water molecules. $\mathrm{Ni}(\mathrm{II})$ complex (3) showed an endothermic peak at $50^{\circ} \mathrm{C}$ due to broken of the hydrogen bondings. Another endothermic peak was observed at $80^{\circ} \mathrm{C}$, with $2.38 \%$ weight loss (Calc. $2.53 \%$ ) corresponding to loss of two hydrated water molecules. The loss of coordinated water molecules was accompanied by three endothermic peaks at 120,135 , and $155{ }^{\circ} \mathrm{C}$ with weight losses $2.72 \%(\mathrm{Calc}$. $2.59 \%$ ), $4.1 \%$ (Calc. 3.99\%) and $4.33 \%$ (Calc. 4.16\%) which were assigned to removal of two, three and three coordinated water molecules respectively. The endothermic peak observed at $230^{\circ} \mathrm{C}$, with $8.16 \%$ weight loss (Calc. $8.36 \%$ ) is due to loss of one terminal coordinated $\mathrm{SO}_{4}$ group, whereas, the loss of the other terminal coordinated $\mathrm{SO}_{4}$ group was accompanied by an endothermic peak at $250^{\circ} \mathrm{Cw}$ ith $8.84 \%$ weight loss (Calc. $9.13 \%$ ). The endothermic peak observed at $315^{\circ} \mathrm{C}$, is corresponding to the melting point of the complex. Finally, the complex shows multiple exothermic peaks at 370 , $390,420,450$ and $500^{\circ} \mathrm{C}$, with total $23.13 \%$ weight loss (Calc. $23.56 \%$ ) corresponding to the thermal decomposition of the complexes with the eventually formation of three $\mathrm{NiO}$ molecules. $\mathrm{Ni}$ (II) complex (4) thermogram showed an endothermic peak at $45^{\circ} \mathrm{C}$ due to broken of the hydrogen bondings. An endothermic peak was observed at $80^{\circ} \mathrm{C}$, with $6.33 \%$ weight loss (Calc. 6.35\%) corresponding to loss of two hydrated water molecules. The endothermic peak observed at $150^{\circ} \mathrm{C}$, with $6.69 \%$ weight loss (Calc. $6.78 \%$ ) is due to loss of two coordinated water molecules. The endothermic peak observed at $360^{\circ} \mathrm{C}$, is corresponding to melting point of the complex. Finally, the complex showed multiple exothermic peaks at 405 , $450,485,510$ and $530^{\circ} \mathrm{C}$, with total $14.72 \%$ weight loss (Calc. $15.0 \%$ ) corresponding to thermal decomposition with eventually formation of one $\mathrm{NiO}$ molecule. The thermogram of $\mathrm{Fe}(\mathrm{II})$ complex (8) showed an endothermic peak at $50^{\circ} \mathrm{C}$, due to broken of the hydrogen bondings. An endothermic peak was observed at $85^{\circ} \mathrm{C}$, with $1.41 \%$ weight losses (Calc. $1.29 \%$ ) corresponding to loss of hydrated water molecule. The loss of coordinated water molecules was accompanied by three endothermic peaks at 115,125 , and $155{ }^{\circ} \mathrm{C}$ with weight losses $2.81 \%$ (Calc. $\left.2.61 \%\right), 4.78 \%$ (Calc. $4.02 \%$ ) and $4.57 \%$ (Calc. $4.19 \%$ ) which were assigned to removal of two middle, three terminal and three terminal coordinated water molecules respectively. The endothermic peak observed at $250^{\circ} \mathrm{C}$ with $7.74 \%$ weight loss (Calc. $7.77 \%$ ), was assigned to loss of one terminal coordinated $\mathrm{SO}_{4}$ group. The loss of other terminal coordinated $\mathrm{SO}_{4}$ group was accompanied with an endothermic peak at $300^{\circ} \mathrm{C}$ with $8.45 \%$ weight loss (Calc. $8.43 \%$ ). An endothermic peak was observed at $325^{\circ} \mathrm{C}$ which could be assigned to the melting point. Finally, the complex shows multiple exothermic peaks at 350, 380, 450, 500 and $610^{\circ} \mathrm{C}$, with total $21.83 \%$ weight loss (Calc. $21.95 \%$ ) corresponding to thermal decomposition with the formation of $\mathrm{Fe}_{3} \mathrm{O}_{4}$ molecule. The thermogram of $\mathrm{Cu}(\mathrm{II})$ complex (12) showed an endothermic peak at $45^{\circ} \mathrm{Cdue}$ to broken of hydrogen bondings. The endothermic peak observed at $78^{\circ} \mathrm{C}$, with $5.63 \%$ weight loss (Calc. $5.83 \%$ ) was assigned to loss of two hydrated water molecules. Whereas the endothermic peak observed at $165^{\circ} \mathrm{C}$, with $3.52 \%$ weight loss $(\mathrm{Calc}$. $3.10 \%)$ was ascribed to loss of a coordinated water molecule. Another endothermic peak was observed at $235^{\circ} \mathrm{C}$, with $11.27 \%$ weight loss (Calc. $11.01 \%$ ), which is assigned to loss of coordinated $\mathrm{NO}_{3}$ group. The endothermic peak observed at $330^{\circ} \mathrm{C}$, is corresponding to the melting point of the complex. The complex showed multiple exothermic peaks at 370, 410,435 and $500^{\circ} \mathrm{C}$, with total $13.73 \%$ weight loss (Calc. $14.03 \%$ ) corresponding to thermal decomposition with the final formation of one $\mathrm{CuO}$ molecule. The thermogram of Co(II) complex (13) showed endothermic peak at $45^{\circ} \mathrm{C}$, due to broken of hydrogen bondings. Two endothermic peaks observed at $80^{\circ} \mathrm{C}$ and $90^{\circ} \mathrm{C}$ with $3.52 \%$ weight loss (Calc. $3.38 \%$ ), corresponding to loss of two hydrated water molecules. The loss of two coordinated water molecules was accompanied by an endothermic peak observed at $130^{\circ} \mathrm{C}$, with $3.24 \%$ weight loss (Calc. $3.49 \%$ ). The melting point of the complex appears at $325^{\circ} \mathrm{C}$ as an endothermic peak. Multiple exothermic peaks were observed at $365,450,550$ and $600^{\circ} \mathrm{C}$ with total $7.74 \%$ weight loss (Calc. $7.54 \%$ ) due to thermal decomposition of the complex with the final formation of one CoO molecule. The thermogram of $\mathrm{Zn}$ (II) complex (15) showed an endothermic peak at $45^{\circ} \mathrm{C}$, corresponding to broken of hydrogen bondings, whereas, the endothermic peak observed at $70^{\circ} \mathrm{C}$, with $2.46 \%$ weight loss (Calc. $2.73 \%$ ) was assigned to loss of two hydrated water molecules. The loss of two acetate groups was accompanied by two endothermic peaks at 210 and $225{ }^{\circ} \mathrm{C}$ with $18.33 \%$ weight loss (Calc. $18.97 \%$ ). An endothermic peak was observed at $345^{\circ} \mathrm{C}$, corresponding to the melting point of the complex. Finally, multiple exothermic peaks was observed at $370,390,410,450$ and $510^{\circ} \mathrm{C}$, with total $16.67 \%$ weight loss (Calc. 16.1\%), assigned to thermal decomposition process with the formation of one $\mathrm{ZnO}$ molecule. The thermogram of $\mathrm{Cu}(\mathrm{II})$ complex (16) showed an endothermic peak at $50^{\circ} \mathrm{Cdue}$ to broken of hydrogen bondings, whereas the loss of one hydrated water molecule was accompanied with endothermic peak at $90^{\circ} \mathrm{C}$ with $1.41 \%$ weight loss (Calc. $1.27 \%$ ). The loss of coordinated water molecules was accompanied by three endothermic peaks at 130 , 150 , and $170{ }^{\circ} \mathrm{C}$ with weight losses $2.13 \%$ (Calc. 2.57\%), 4.25\% (Calc. 3.95\%) and 3.83\% (Calc. 4.11\%) which were assigned to removal of two middle, three terminal and three terminal coordinated water molecules respectively. An endothermic peak was observed at $215^{\circ} \mathrm{C}$ with $7.80 \%$ weight loss (Calc. $7.63 \%$ ) which could be assigned to loss of a coordinated $\mathrm{SO}_{4}$ group, whereas, the loss the other sulfate group was accompanied with an endothermic peak at $230^{\circ} \mathrm{C}$ with $8.51 \%$ weight loss (Calc. $8.26 \%$ ). The endothermic peak observed at $360^{\circ} \mathrm{C}$ was assigned to the melting point of the complex. Thermal decomposition of the complex was accompanied by multiple exothermic peaks at $390,430,510,530$ and $630^{\circ} \mathrm{C}$ with total $21.98 \%$ weight loss (Calc. 22.23\%) with final formation of three $\mathrm{CuO}$ molecules. 


\section{《[(4)}

Table 5:- ESR data for metal (II) complexes

\section{ISSN 2321-807X}

\begin{tabular}{|c|c|c|c|c|c|c|c|c|c|c|c|c|c|c|c|c|c|c|c|}
\hline Complex & g & g & $\mathrm{g}_{\text {iso }} \mathrm{a}^{\mathrm{a}}$ & $\begin{array}{c}\text { A } \\
\text { (G) }\end{array}$ & $\begin{array}{c}\mathbf{A} \\
\text { (G) }\end{array}$ & $\begin{array}{c}\mathbf{A}_{\text {is o }}^{\mathbf{b}} \\
\text { (G) }\end{array}$ & $\mathbf{G}^{\mathrm{c}}$ & $\begin{array}{l}\Delta \mathbf{E}_{\mathrm{xy}} \\
\left(\mathrm{cm}^{-1}\right)\end{array}$ & $\begin{array}{c}\Delta \mathbf{E}_{\mathrm{xz}} \\
\left(\mathrm{cm}^{-1}\right)\end{array}$ & $\mathbf{K}^{2}$ & $\mathbf{K}^{2}$ & $\mathbf{K}^{2}$ & $\mathbf{K}$ & $\begin{array}{c}\mathrm{g} / \mathrm{A} \\
\left(\mathrm{cm}^{-1}\right)\end{array}$ & $\alpha^{2}$ & $\beta^{2}$ & $\beta_{1}^{2}$ & $-2 \beta$ & $\mathbf{a}^{2} \mathbf{d}(\%)$ \\
\hline (2) & - & - & 2.13 & $\begin{array}{l}- \\
-\end{array}$ & - & - & $\begin{array}{lll}- & -\end{array}$ & - & - & - & - & & - & - & 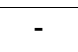 & - & - & - & - \\
\hline (5) & 2.26 & 2.04 & 2.11 & 130 & 10 & 50 & 6.5 & 17094 & 21505 & 0.49 & 0.66 & 0.54 & 0.73 & 165 & 0.68 & 0.72 & 0.97 & 190.5 & 81 \\
\hline (7) & 2.22 & 2.07 & 2.12 & 180 & 15 & 70 & 3.14 & 17391 & 22988 & 0.71 & 0.75 & 0.74 & 0.86 & 123.3 & 0.88 & 0.80 & 0.85 & 126.6 & 54 \\
\hline (8) & - & - & 2.09 & - & - & - & - & - & - & - & - & - & - & - & - & - & - & - & - \\
\hline (10) & - & - & 2.01 & - & - & - & - & - & - & - & - & - & - & - & - & - & - & - & - \\
\hline (12) & 2.71 & 2.06 & 2.09 & 150 & 15 & 60 & 2.83 & 16949 & 21505 & 0.75 & 0.43 & 0.64 & 0.8 & 144.7 & 0.65 & 1.15 & 0.66 & 162.8 & 69.3 \\
\hline (13) & - & - & 2.09 & - & - & - & - & - & - & - & - & - & - & - & - & - & - & - & - \\
\hline (16) & 2.18 & 2.05 & 2.09 & 125 & 12.5 & 50 & 3.6 & 16949 & 21505 & 0.62 & 0.45 & 0.56 & 0.75 & 171.6 & 0.59 & 1.05 & 0.76 & 127.1 & 54 \\
\hline
\end{tabular}

a) $3 g_{\text {iso }}=g+2 g \perp$

b) $3 A_{\text {iso }}=A+2 A \perp$

c) $G=g-2 / g \perp-2$ 
Table 6:- Thermal analyses for metal (II) complexes

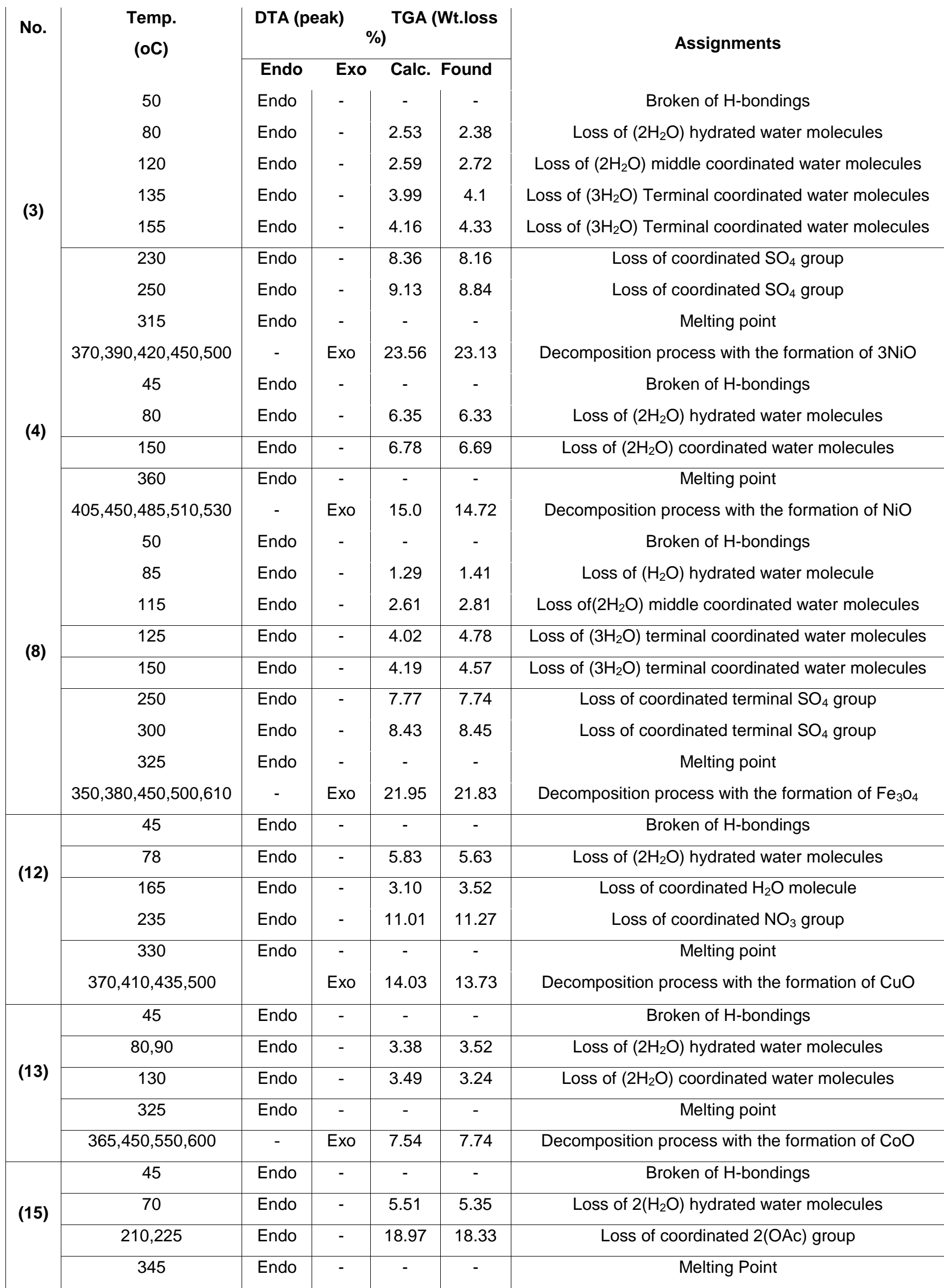




\begin{tabular}{|c|c|c|c|c|c|c|}
\hline & $370,390,410,450,510$ & - & Exo & 16.1 & 16.67 & Decomposition process with the formation of $\mathrm{ZnO}$ \\
\hline & 45 & Endo & - & - & - & Broken of $\mathrm{H}$-bondings \\
\hline \multirow{9}{*}{ (16) } & 50 & Endo & - & - & - & Broken of $\mathrm{H}$-bondings \\
\hline & 90 & Endo & - & 1.27 & 1.41 & Loss of hydrated $\mathrm{H}_{2} \mathrm{O}$ \\
\hline & 130 & Endo & - & 2.57 & 2.13 & Loss of $\left(2 \mathrm{H}_{2} \mathrm{O}\right)$ middle coordinated water molecules \\
\hline & 150 & Endo & - & 3.95 & 4.25 & Loss of $\left(3 \mathrm{H}_{2} \mathrm{O}\right)$ terminal coordinated water molecules \\
\hline & 170 & Endo & - & 4.11 & 3.83 & Loss of $\left(3 \mathrm{H}_{2} \mathrm{O}\right)$ terminal coordinated water molecules \\
\hline & 215 & Endo & - & 7.63 & 7.80 & Loss of coordinated $\mathrm{SO}_{4}$ group \\
\hline & 230 & Endo & - & 8.26 & 8.51 & Loss of coordinated $\mathrm{SO}_{4}$ group \\
\hline & 360 & Endo & - & - & - & Melting Point \\
\hline & $\begin{array}{c}390,430,510,530 \\
630\end{array}$ & - & - & 22.23 & 21.98 & Decomposition process with the formation of $3 \mathrm{CuO}$ \\
\hline
\end{tabular}

\subsection{Biological studies}

\subsubsection{Cytotoxic activity}

The ligand and some metal complexes were evaluated for their cytotoxicity against two different tumor cell lines (HEP. G2 and HCT-116) by MTT assay using Vinblastine as a standard drug. It is interesting to note that, the selected compounds showed cytotoxicity potential in the range of cancerous cell lines tested (Figure 3). The $\mathrm{IC}_{50}$ values derived from the experimental data were summarized in table 7. It was reported that, compounds exhibiting $I_{50}$ values more than $10-25 \mu \mathrm{g} / \mathrm{ml}$ are treated as weak cytotoxic activities while compounds with $I_{50}$ values less than $5 \mu \mathrm{g} / \mathrm{ml}$ are considered to be very active. Those having intermediate values ranging from 5 to $10 \mu \mathrm{g} / \mathrm{ml}$ are classified as moderately active [83]. The invitro cytotoxicity values demonstrated that, the tested complexes have higher activity in comparison with that of the ligand against (HCT-116) tumor cell lines. Cu(II) complex (5) demonstrated very active cytotoxicity with $\mathrm{IC}_{50}$ values 2.76 $\mu \mathrm{g} / \mathrm{ml}$, whereas $\mathrm{Ni}(\mathrm{II})$ complex (4) showed moderate cytotoxicity with $\mathrm{IC}_{50}$ values $12.2 \mu \mathrm{g} / \mathrm{ml}$, in the time that the ligand (1) recorded weak cytotoxicity with $\left(\mathrm{IC}_{50}\right.$ values $\left.20.1 \mu \mathrm{g} / \mathrm{ml}\right)$ comparing with the control. The enhancement of cytotoxic activity may be assigned to that the positive charge of the metal increases the acidity of coordinated ligand that bears protons, leading to stronger hydrogen bonds and enhancement of the biological activity [84,85]. It was shown also that, there is a positive correlation between the surviving fraction ratio of tumor cell lines and the metal complexesconcentrations. The biological assays of the metal complexes against (HEP-G2) tumor cell lines revealed that, Zn(II) complex (15) exhibits the highest inhibitory ability with $\mathrm{IC}_{50}$ value equals $5.26 \mu \mathrm{g} / \mathrm{ml}$. This value is slightly higher when compared with complex (14) $\left(\mathrm{IC}_{50} 6.13 \mu \mathrm{g} / \mathrm{ml}\right)$. On the other hand $\mathrm{Co}(\mathrm{II})$ complex (2) recorded a weak cytotoxicity with $\left(\mathrm{IC}_{50} \mathrm{values} 24.6 \mu \mathrm{g} / \mathrm{ml}\right) \mathrm{in}$ comparison with the control drug. These findings suggest that both cupper(II) complex (5) and Zn(II) complex(15) exhibit promising potentials as an anticancer compounds against (HEP-G2andHCT-116) tumor cell respectively. (Figure 4, 5)

Table 7. Cytotoxic activity (IC50) of selected metal complexes against human colon carcinoma cells (HCT-116 cell line) and hepatocellular carcinoma cells (HEPG-2 cell line).

\begin{tabular}{|c|c|c|c|}
\hline \multirow{2}{*}{ No. } & \multirow{2}{*}{ Compound } & \multicolumn{2}{|l|}{$\mathrm{IC}_{50}(\mu \mathrm{g} / \mathrm{ml})$} \\
\hline & & HEPG-2 & HCT-116 \\
\hline (1) & {$\left[\mathrm{H}_{2} \mathrm{~L}\right]$} & 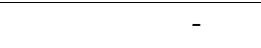 & 20.1 \\
\hline (2) & {$\left[(\mathrm{L}) \mathrm{Co}\left(\mathrm{H}_{2} \mathrm{O}\right)_{2}\right] \cdot \mathrm{H}_{2} \mathrm{O}$} & 24.6 & - \\
\hline (4) & {$\left[(\mathrm{L}) \mathrm{Ni}\left(\mathrm{H}_{2} \mathrm{O}\right)_{2}\right] \cdot 2 \mathrm{H}_{2} \mathrm{O}$} & - & 12.2 \\
\hline (5) & {$\left[(\mathrm{L}) \mathrm{Cu}\left(\mathrm{H}_{2} \mathrm{O}\right)_{2}\right] \cdot 2 \mathrm{H}_{2} \mathrm{O}$} & - & 2.76 \\
\hline (14) & {$\left[(\mathrm{HL})_{2} \mathrm{Cd}(\mathrm{OAc})_{2}\right] \cdot 2 \mathrm{H}_{2} \mathrm{O}$} & 6.13 & - \\
\hline (15) & {$\left[\left(\mathrm{H}_{2} \mathrm{~L}\right) \mathrm{Zn}(\mathrm{OAc})_{2}\right] \cdot 2 \mathrm{H}_{2} \mathrm{O}$} & 5.26 & - \\
\hline Standard & & 4.6 & 2.38 \\
\hline
\end{tabular}




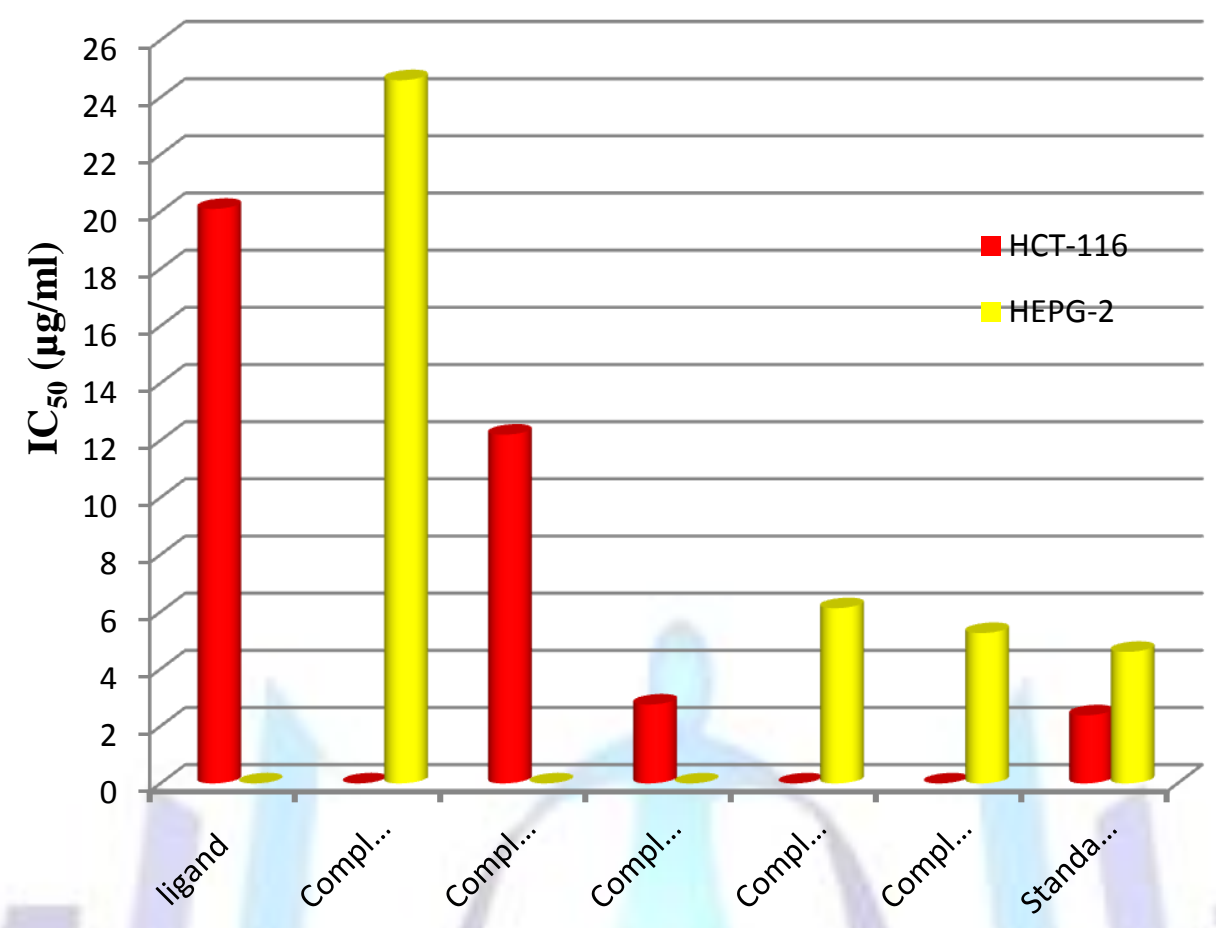

Figure 3: $\mathrm{IC}_{50}$ values of the ligand $\left[\mathrm{H}_{2} \mathrm{~L}\right]$ and some metal complexes against human hepatocarcinoma (HEPG-2) and human colon cancer cell lines (HCT-116.)

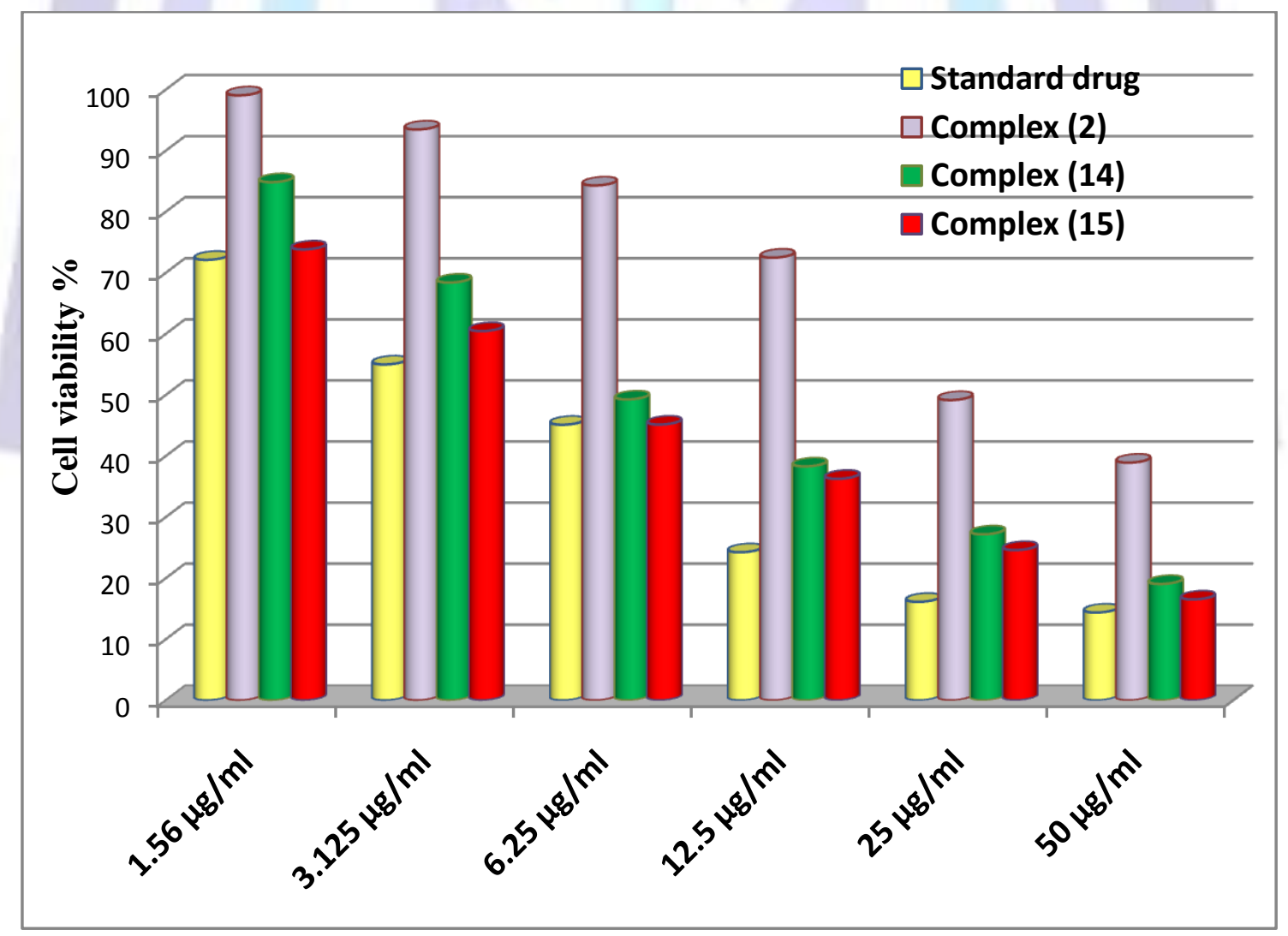

Figure 4: Antiproliferative activity against human hepatocarcinoma (HEPG-2.) at different metal complexes concentrations. 


\section{ISSN 2321-807X}

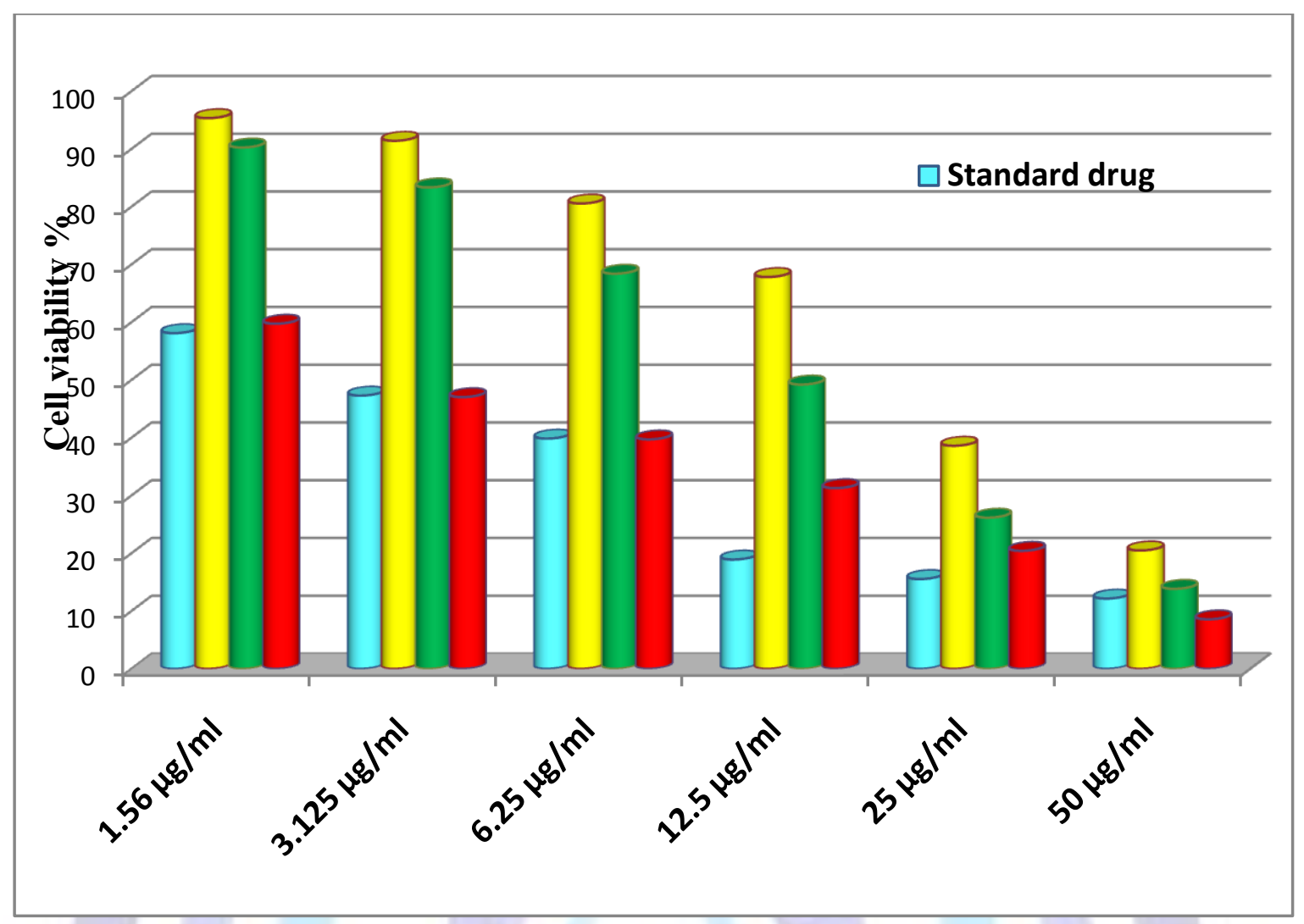

Figure 4.Antiproliferative activity against human colon cell lines (MCT-116) at different the metal complexes concentrations.

\section{Conclusion.}

The newly synthesized Schiff base ligand derived from (1E, N'Z, N'Z)-N', N'-bis (2-hydroxybenzylidene)-2(naphthalen-1-yloxy) acetohydrazonohydrazide $\left[\mathrm{H}_{2} \mathrm{~L}\right]$ act as bidentate or a tetradentatetridentate ligands, and in all complexes was coordinated through the azomethine nitrogen and phenolic oxygen groups to the metal ion. All the synthesized metal(II) complexes possessed an octahedral geometry except the sliver complex (6), copper complex (7) and cobalt complexes (11). The reasonable agreement between the theoretical and experimental data reflects to the great extent the suitability of the suggested structures. The invitro cytotoxicity values demonstrated that the tested complexes have higher activity than the ligand against (HCT-116). Cupper(II) complex (5) and Zn(II) complex (15) exhibit promising potentials as an anticancer compounds against (HCT-116 and HEP-G2) tumor cell respectively.

\section{References}

[1]Radunsky, C., Kösters J., Müller J. 2015. Chromogenic behaviour of a family of hydrazine and hydrazone metal complexes.InorganicaChimicaActa 428 (0):14-20.

[2]Premkumar, T., Govindarajan S. 2002. The chemistry of hydrazine derivatives-thermal behavior and characterisation of hydrazinium salts and metal hydrazine complexes of 4,5-imidazoledicarboxylic acid. ThermochimicaActa 386 (1):35-42.

[3]Heaton, B. T., Jacob C., Page P. 1996. Transition metal complexes containing hydrazine and substituted hydrazines. Coordination Chemistry Reviews 154 (0):193-229.

[4]Prakash, G., Manikandan R., Viswanathamurthi P., Velmurugan K., Nandhakumar R. 2014. Ruthenium(III) Smethylisothiosemicarbazone Schiff base complexes bearing PPh3/AsPh3 coligand: Synthesis, structure and biological investigations, including antioxidant, DNA and protein interaction, and in vitro anticancer activities. Journal of Photochemistry and Photobiology B: Biology $138(0): 63-74$.

[5]Li, X., Bi C.-f., Fan Y.-h., Zhang X., Meng X.-m., Cui L.-s. 2014. Synthesis, crystal structure and anticancer activity of a novel ternary copper(II) complex with Schiff base derived from 2-amino-4-fluorobenzoic acid and salicylaldehyde. Inorganic Chemistry Communications 50 (0):35-41.

[6]Bhat, M. A., Iqbal M., Al-Dhfyan A., Shakeel F. 2015. Carvone Schiff base of isoniazid as a novel antitumor agent: Nanoemulsion development and pharmacokinetic evaluation. Journal of Molecular Liquids 203 (0):111-119. 
[7]El-Tabl, A. S., Shakdofa M. M. E., Whaba M. A. 2015. Synthesis, characterization and fungicidal activity of binary and ternary metal(II) complexes derived from 4,4'-((4-nitro-1,2-phenylene) bis(azanylylidene))bis(3-(hydroxyimino)pentan2-one). SpectrochimicaActa Part A: Molecular and Biomolecular Spectroscopy 136, Part C (0):1941-1949.

[8]A. S. Eltabl, M. A.-E., M. A. Wahba, S. A. EL-assaly, L. M. Saad 2014.Sugar Hydrazone Complexes; Synthesis, Spectroscopic Characterization and Antitumor Activity. Journal of Advances in Chemistry 9 (1):1837-1860.

[9]Sun, R. W.-Y., Ma D.-L., Wong E. L.-M., Che C.-M. 2007. Some uses of transition metal complexes as anti-cancer and anti-HIV agents. Dalton transactions (43):4884-4892.

[10]Haas, K. L., Franz K. J. 2009. Application of metal coordination chemistry to explore and manipulate cell biology. Chemical reviews 109 (10):4921-4960.

[11]Correia I1, A. P., Roy S1, Wahba M2, Matos C1, Maurya MR3, Marques F4, Pavan FR5, Leite CQ5, Avecilla F6, Costa Pessoa J7. 2014. Hydroxyquinoline derived vanadium(IV and V) and copper(II) complexes as potential antituberculosis and anti-tumor agents. J InorgBiochem 41 (C):83-93.

[12]Correia, I., Adão P., Roy S., Wahba M., Matos C., Maurya M. R., Marques F., Pavan F. R., Leite C. Q. F., Avecilla F., Costa Pessoa J. 2014. Hydroxyquinoline derived vanadium(IV and V) and copper(II) complexes as potential antituberculosis and anti-tumor agents. Journal of Inorganic Biochemistry $141(0): 83-93$.

[13]Fricker, S. P. 2007. Metal based drugs: from serendipity to design. Dalton transactions (43):4903-4917.

[14]Meggers, E. 2009.Targeting proteins with metal complexes. Chemical Communications (9):1001-1010.

[15]Shchepin, R., Navarathna D. H. M. L. P., Dumitru R., Lippold S., Nickerson K. W., Dussault P. H. 2008. Influence of heterocyclic and oxime-containing farnesol analogs on quorum sensing and pathogenicity in Candida albicans. Bioorganic \& Medicinal Chemistry 16 (4):1842-1848.

[16]Chandra, S., Vandana, Kumar S. 2015. Synthesis, spectroscopic, anticancer, antibacterial and antifungal studies of $\mathrm{Ni}(\mathrm{II})$ and $\mathrm{Cu}$ (II) complexes with hydrazine carboxamide, 2-[3-methyl-2-thienyl methylene]. SpectrochimicaActa Part A: Molecular and Biomolecular Spectroscopy 135 (0):356-363.

[17]Liang, J.-H., Lv W., Li X.-L., An K., Cushman M., Wang H., Xu Y.-C. 2013. Synthesis and antibacterial activity of 9 oxime ether non-ketolides, and novel binding mode of alkylides with bacterial rRNA. Bioorganic \& Medicinal Chemistry Letters 23 (5):1387-1393.

[18]El-Gamal, M. I., Bayomi S. M., El-Ashry S. M., Said S. A., Abdel-Aziz A. A. M., Abdel-Aziz N. I. 2010. Synthesis and anti-inflammatory activity of novel (substituted)benzylidene acetone oxime ether derivatives: Molecular modeling study. European Journal of Medicinal Chemistry 45 (4):1403-1414.

[19]Nirmala, P., Ramanathan M. 2011. Effect of kaempferol on lipid peroxidation and antioxidant status in 1,2-dimethyl hydrazine induced colorectal carcinoma in rats. European Journal of Pharmacology 654 (1):75-79.

[20]Özyürek, M., Akpınar D., Bener M., Türkkan B., Güçlü K., Apak R. 2014. Novel oxime based flavanone, naringinoxime: Synthesis, characterization and screening for antioxidant activity. Chemico-Biological Interactions 212 (0):4046.

[21]Wang, R., Zhang X., Song H., Zhou S., Li S. 2014. Synthesis and evaluation of novel alkannin and shikonin oxime derivatives as potent antitumor agents. Bioorganic \& Medicinal Chemistry Letters 24 (17):4304-4307.

[22]Dilman, V. M., Anisimov V. N. 1979. Potentiation of antitumor effect of cyclophosphamide and hydrazine sulfate by treatment with the antidiabetic agent, 1-phenylethylbiguanide (phenformin). Cancer Letters 7 (6):357-361.

[23]Ma, J., Chen D., Lu K., Wang L., Han X., Zhao Y., Gong P. 2014. Design, synthesis, and structure-activity relationships of novel benzothiazole derivatives bearing the ortho-hydroxy $\mathrm{N}$-carbamoylhydrazone moiety as potent antitumor agents. European Journal of Medicinal Chemistry 86 (0):257-269.

[24]Dai, H., Xiao Y.-S., Li Z., Xu X.-Y., Qian X.-H. 2014. The thiazoylmethoxy modification on pyrazole oximes: Synthesis and insecticidal biological evaluation beyond acaricidal activity. Chinese Chemical Letters 25 (7):1014-1016.

[25]Ramadan M.El-Bahnasawy1 , F. A. E.-S., EimanH.A.Gaafer 1, Mohammed A.Wahba 2013. Benzilbisisonicotinoylhydrazone complexes of tervalent metals Ti, Zr, Sn, Hf and Th. ICAIJ 8 ((1)):1-6.

[26]Archer, C. M., Dilworth J. R., Jobanputra P., Thompson R. M., McPartlin M., Povey D. C., Smith G. W., Kelly J. D. 1990. Development of new technetium cores containing technetium-nitrogen multiple bonds.Synthesis and characterization of some diazenido-, hydrazido- and imido- complexes of technetium. Polyhedron 9 (12):1497-1502.

[27]Carrillo, D. 2000. [MoO2(acac)2], a versatile precursor for diazenido- and hydrazido-complexes. ComptesRendus de I'Académie des Sciences - Series IIC - Chemistry 3 (3):175-181.

[28]Riveros, P. C., Perilla I. C., Poveda A., Keller H. J., Pritzkow H. 2000. Tris(dialkyldithiocarbamato)diazenido(1-) and hydrazido(2-) molybdenum complexes: synthesis and reactivity in acid medium. Polyhedron 19 (22-23):2327-2335.

[29]Svehla, G. (1979) Vogel's Textbook of macro and semimicro qualitative inorganic analysis. Longman London,

[30]Welcher, F. J. 1958.analytical uses of ethylenediaminetetraacetic acid. 


\section{ISSN 2321-807X}

[31]Vogel, A.A Text Book of Quantitative Inorganic Analysis, 1978. ELBS, London:474.

[32]Figgis, B., Lewis J., Wilkins R. 1960. Modern coordination chemistry.Interscience, New York:403.

[33]El-Tabl, A. S., El-Enein S. A. 2004.Reactivity of the new potentially binucleating ligand, 2-(acetichydrazido-Nmethylidene-a-naphthol)-benzothiazol, towards manganese (II), nickel (II), cobalt (II), copper (II) and zinc (II) salts. Journal of Coordination Chemistry 57 (4):281-294.

[34]Nicodemo, A., Araujo M., Ruiz A., Gales A. 2004. In vitro susceptibility of Stenotrophomonasmaltophilia isolates: comparison of disc diffusion, Etest and agar dilution methods. Journal of Antimicrobial Chemotherapy 53 (4):604-608.

[35]Standards, N. C. f. C. L. (2001) Performance standards for antimicrobial disk and dilution susceptibility tests for bacteria isolated from animals. Approved standard. Approved standard.,vol M31-A2. NCCLS, Wayne, Pennsylvania

[36]J.G. Collee, J.P. Duguid, A.G. Farser, B.D. Marmion e. (eds) (1989) Practical medical microbiolog. Churchill Livingstone,, New York,

[37]Gup, R., Kırkan B. 2005. Synthesis and spectroscopic studies of copper (II) and nickel (II) complexes containing hydrazonic ligands and heterocyclic coligand. SpectrochimicaActa Part A: Molecular and Biomolecular Spectroscopy $62(4): 1188-1195$.

[38]El-Tabl, A. S., Shakdofa M. M., El-Seidy A. M., Al-Hakimi A. N. 2012. Synthesis, characterization and antifungal activity of metal complexes of 2-(5-((2-chlorophenyl) diazenyl)-2-hydroxybenzylidene) hydrazinecarbothioamide. Phosphorus, Sulfur, and Silicon and the Related Elements 187 (11):1312-1323.

[39]Raman, N., Muthuraj V., Ravichandran S., Kulandaisamy A. 2003. Synthesis, characterisation and electrochemical behaviour of $\mathrm{Cu}$ (II), $\mathrm{Co}$ (II), $\mathrm{Ni}$ (II) and $\mathrm{Zn}$ (II) complexes derived from acetylacetoneandp-anisidine and their antimicrobial activity. Journal of Chemical sciences 115 (3):161-167.

[40]Mendíl, D., Bílgín A., Gök Y., Şentük H. B. 2002. Synthesis and characterization of a new (E, E)-dioxime and its homo and heteronuclear complexes containing macrobicylic moieties. Journal of inclusion phenomena and macrocyclic chemistry 43 (3-4):265-270.

[41]Tircsó, G., Bényei A., Brücher E., Kis A., Király R. 2006. Equilibria and structure of the lanthanide (III)-2-hydroxy-1, 3diaminopropane-N, N, N', N'-tetraacetate complexes: Formation of alkoxo-bridged dimers in solid state and solution. Inorganic chemistry 45 (13):4951-4962.

[42]Geraldes, C. F., Marques M. P. M., Sherry A. D. 1998. NMR conformational study of diamagnetic complexes of some triazatriacetate macrocycles. Inorganicachimicaacta 273 (1):288-298.

[43]Caminiti, R., Cucca P., Monduzzi M., Saba G., Crisponi G. 1984.Divalent metal-acetate complexes in concentrated aqueous solutions. An x-ray diffraction and NMR spectroscopy study. The Journal of chemical physics 81 (1):543551.

[44]Daumann, L. J., Schenk G., Ollis D. L., Gahan L. R. 2014. Spectroscopic and mechanistic studies of dinuclearmetallohydrolases and their biomimetic complexes. Dalton Transactions 43 (3):910-928.

[45]Deveci, P., Taner B., Kılıç Z., Solak A. O., Arslan U., Özcan E. 2011. Novel redox-active macrocyclic vic-dioxime ligand and its metal complexes modified with azacrown ether: Spectral, cyclic voltammetric and antimicrobial activity studies. Polyhedron 30:1726-1731.

[46]Pavia, D., Lampman G., Kriz G., Vyvyan J. 2009. Introduction to Spectroscopy Cengage Learning. Belmont, CA, USA.

[47]Naskar, S., Naskar S., Mondal S., Majhi P. K., Drew M. G., Chattopadhyay S. K. 2011. Synthesis and spectroscopic properties of cobalt (III) complexes of some aroylhydrazones: X-ray crystal structures of one cobalt (III) complex and two aroylhydrazone ligands. Inorganicachimicaacta 371 (1):100-106.

[48]Gulaczyk, I., Kręglewski M. 2008. The symmetric amino-wagging band of hydrazine: Assignment and analysis. Journal of Molecular Spectroscopy 249 (2):73-77.

[49]Gulaczyk, I., Kręglewski M., Valentin A. 2003. The N-N stretching band of hydrazine. Journal of Molecular Spectroscopy $220(1): 132-136$.

[50]Singh G, Singh P, Singh K, Singh D, Handa R, Dubey N 2002. Proc Natl AcadScilnd 72A:87.

[51]Monfared, H. H., Kalantari Z., Kamyabi M. A., Janiak C. 2007. Synthesis, Structural Characterization and Electrochemical Studies of a Nicotinamide-bridged Dinuclear Copper Complex derived from a Tridentate Hydrazone Schiff Base Ligand. Zeitschriftfüranorganische und allgemeineChemie 633 (11-12):1945-1948.

[52]Pouralimardan, O., Chamayou A.-C., Janiak C., Hosseini-Monfared H. 2007. Hydrazone Schiff base-manganese (II) complexes: Synthesis, crystal structure and catalytic reactivity. InorganicaChimicaActa 360 (5):1599-1608.

[53]Kannan, S., Ramesh R. 2006. Synthesis, characterization, catalytic oxidation and biological activity of ruthenium (III) Schiff base complexes derived from 3-acetyl-6-methyl-2H-pyran-2, 4 (3H)-dione. Polyhedron 25 (16):3095-3103.

[54]Lin-Vien, D., Colthup N. B., Fateley W. G., Grasselli J. G. (1991) The handbook of infrared and Raman characteristic frequencies of organic molecules. Elsevier, 
[55]El-Tabl, A. S., Plass W., Buchholz A., Shakdofa M. M. 2009.Synthesis, spectroscopic investigation and biological activity of metal (II) complexes with N 204 ligands. Journal of Chemical Research 2009 (9):582-587.

[56]Keskioğlu, E., Gündüzalp A. B., Cete S., Hamurcu F., Erk B. 2008. Cr (III), Fe (III) and Co (III) complexes of tetradentate (ONNO) Schiff base ligands: synthesis, characterization, properties and biological activity. SpectrochimicaActa Part A: Molecular and Biomolecular Spectroscopy 70 (3):634-640.

[57]Teotia, M., Gurtu J., Rana V. 1980.Dimeric 5-and 6-coordinate complexes of tri and tetradentate ligands. Journal of Inorganic and Nuclear Chemistry 42 (6):821-831.

[58]Fouda, M., Abd-Elzaher M., Shakdofa M., El Saied F., Ayad M., El Tabl A. 2008.Synthesis and characterization of transition metal complexes of $\mathrm{N}^{\prime}$-[(1, 5-dimethyl-3-oxo-2-phenyl-2, 3-dihydro-1H-pyrazol-4-yl) methylene] thiophene-2carbohydrazide. Transition Metal Chemistry 33 (2):219-228.

[59]Nakamoto, K. (1978) Infrared and Raman spectra of inorganic and coordination compounds. Wiley Online Library,

[60]Murukan, B., Mohanan K. 2006. Synthesis, Characterization, Electrochemical Properties and Antibacterial Activity of Some Transiton Metal Complexes with [(2-hydroxy-1-naphthaldehyde)-3-isatin]-bishydrazone. Transition Metal Chemistry 31 (4):441-446.

[61]El-Tabl, A. S., El-Saied F. A., Al-Hakimi A. N. 2007.Synthesis, spectroscopic investigation and biological activity of metal complexes with ONO trifunctionalalizedhydrazone ligand. Transition Metal Chemistry 32 (6):689-701.

[62]K, N. (1967) Infrared spectra of inorganic and coordination compounds 2nd edn, Wiley Inc. , .2nd ednedn. Wiley Inc., New York

[63]Steed, J. W., Tocher D. A. 1994.Nitrato complexes of ruthenium (IV): chelating,"semi-chelating" and monodentate coordination modes. Polyhedron 13 (2):167-173.

[64]Nakamoto, K. (1986) Infrared and Raman spectra of inorganic and coordination compounds. Wiley Online Library,

[65]Fouda, M. F., Abd-Elzaher M. M., Shakdofa M. M., El-Saied F. A., Ayad M. I., El Tabl A. S. 2008. Synthesis and characterization of a hydrazone ligand containing antipyrine and its transition metal complexes. Journal of Coordination Chemistry 61 (12):1983-1996.

[66]Lever, A. 1968. Electronic spectra of some transition metal complexes: Derivation of Dq and B. Journal of Chemical Education 45 (11):711.

[67]Aslan, H. G., Özcan S., Karacan N. 2011. Synthesis, characterization and antimicrobial activity of salicylaldehydebenzenesulfonylhydrazone (<i>Hsalbsmh</i>) and its Nickel (II), Palladium (II), Platinum (II), Copper (II), Cobalt (II) complexes. Inorganic Chemistry Communications 14 (9):1550-1553.

[68]Mohamed, G. G., Omar M., Hindy A. M. 2005. Synthesis, characterization and biological activity of some transition metals with Schiff base derived from 2-thiophene carboxaldehyde and aminobenzoic acid. SpectrochimicaActa Part A: Molecular and Biomolecular Spectroscopy 62 (4):1140-1150.

[69]Geary, W. J. 1971. The use of conductivity measurements in organic solvents for the characterisation of coordination compounds. Coordination Chemistry Reviews 7 (1):81-122.

[70]Chohan, Z. H., Supuran C. T. 2005. Organometallic compounds with biologically active molecules: in vitro antibacterial and antifungal activity of some 1, 1'-(dicarbohydrazono) ferrocenes and their cobalt (II), copper (II), nickel (II) and zinc (II) complexes. Applied organometallic chemistry 19 (12):1207-1214.

[71]Akbar Ali, M., Mirza A. H., Yee C. Y., Rahgeni H., Bernhardt P. V. 2011. Mixed-ligand ternary complexes of potentially pentadentate but functionally tridentate Schiff base chelates. Polyhedron 30 (3):542-548.

[72]Surati, K. R., Thaker B. 2010. Synthesis, spectral, crystallography and thermal investigations of novel Schiff base complexes of manganese (III) derived from heterocyclic $\beta$-diketone with aromatic and aliphatic diamine. SpectrochimicaActa Part A: Molecular and Biomolecular Spectroscopy 75 (1):235-242.

[73]Surati, K. R. 2011. Synthesis, spectroscopy and biological investigations of manganese (III) Schiff base complexes derived from heterocyclic $\beta$-diketone with various primary amine and 2, 2'-bipyridyl. SpectrochimicaActa Part A: Molecular and Biomolecular Spectroscopy 79 (1):272-277.

[74]Kennedy, B. J., Murray K. S. 1985.Magnetic properties and zero-field splitting in high-spin manganese (III) complexes. 1. Mononuclear and polynuclear Schiff-base chelates. Inorganic Chemistry 24 (10):1552-1557.

[75]Hathaway, B. J., Billing D. E. 1970. The electronic properties and stereochemistry of mono-nuclear complexes of the copper(II) ion. Coordination Chemistry Reviews 5 (2):143-207.

[76]Eisenstein, J. C. 1958. Effect of Exchange Interaction on the Magnetic and Thermal Properties of Copper Salts and Copper Coordination Compounds. The Journal of Chemical Physics 28 (2):323-329.

[77]Tomlinson, A. A. G., Hathaway B. J. 1968. The electronic properties and stereochemistry of the copper(II) ion. Part II. The monoamine adducts of bisethylenediaminecopper(II) complexes. Journal of the Chemical Society A: Inorganic, Physical, Theoretical (0):1685-1688. 
[78]El-Tabl, A. S., Aly F. A., Shakdofa M. M. E., Shakdofa A. M. E. 2010.Synthesis, characterization, and biological activity of metal complexes of azohydrazone ligand. Journal of Coordination Chemistry 63 (4):700-712.

[79]Al-Hakimi, A. N., El-Tabl A. S., Shakdofa M. M. 2009. Coordination and biological behaviour of 2-(p-toluidino)-N'-(3oxo-1, 3-diphenylpropylidene) acetohydrazide and its metal complexes. Journal of Chemical Research 2009 (12).

[80]Shauib, N. M., Elassar A.-Z. A., El-Dissouky A. 2006. Synthesis and spectroscopic characterization of copper (II) complexes with the polydentate chelating ligand 4, 4'-[1, 4-phenylenedi (nitrilo) dipente-2-one.SpectrochimicaActa Part A: Molecular and Biomolecular Spectroscopy 63 (3):714-722.

[81]Greenwood, N. N., Straughan B. P., Wilson A. E. 1968. Behaviour of tellurium(IV) chloride, bromide, and iodide in organic solvents and the structures of the species present. Journal of the Chemical Society A: Inorganic, Physical, Theoretical (0):2209-2212.

[82]Symons, M. C., Trousson P. M. 1984. Electron spin resonance studies of the radiolysis of methyl isocyanate and methyl isothiocyanate. Radiation Physics and Chemistry (1977) 23 (1):127-135.

[83]A. S. Eltabl, M. M. A.-E. W., M. A. Wahba, S. A. EL-assaly, L. M. Saad 2014. Sugar Hydrazone Complexes; Synthesis, Spectroscopic Characterization and Antitumor Activity. Journal of Advances in Chemistry 9 (1):1837-1860.

[84]Feng, G., Mareque-Rivas J. C., Williams N. H. 2006. Comparing a mononuclear Zn (II) complex with hydrogen bond donors with a dinuclear Zn (II) complex for catalysing phosphate ester cleavage. Chemical Communications (17):1845-1847.

[85]Mareque-Rivas, J. C., Prabaharan R., Parsons S. 2004.Quantifying the relative contribution of hydrogen bonding and hydrophobic environments, and coordinating groups, in the zinc (II)-water acidity by synthetic modelling chemistry. Dalton transactions (10):1648-1655.

[86]Tweedy, B. Possible mechanism for reduction of elemental sulfur by moniliniafructicola. In: Phytopathology, 1964. AMER PHYTOPATHOLOGICAL SOC 3340 PILOT KNOB ROAD, ST PAUL, MN 55121, pp 910-\&

[87]Chohan, Z. H., Khan K. M., Supuran C. T. 2005. In-vitro antibacterial, antifungal and cytotoxic properties of sulfonamide-derived Schiff's bases and their metal complexes. Journal of enzyme inhibition and medicinal chemistry $20(2): 183-188$.

[88]Al-Hakimi, A. N., El-Tabl A. S., Shakdofa M. M. 2009. Coordination and biological behaviour of 2-(p-toluidino)-N'-(3oxo-1, 3-diphenylpropylidene) acetohydrazide and its metal complexes. Journal of Chemical Research 2009 (12):770774. 